\title{
An original model of brain infection identifies the hijacking of host lipoprotein import as a bacterial strategy for blood-brain barrier crossing.
}

Billel Benmimoun ${ }^{1}$, Florentia Papastefanaki², Bruno Périchon ${ }^{3}$, Katerina Segklia ${ }^{2}$, Nicolas Roby $^{1}$, Vivi Miriagou ${ }^{4}$, Christine Schmitt ${ }^{5}$, Shaynoor Dramsi ${ }^{3}$, Rebecca Matsas ${ }^{2}$ and Pauline Spéder ${ }^{1, *}$

1. Institut Pasteur, Brain plasticity in response to the environment, CNRS, UMR3738, Paris, France.

2. Hellenic Pasteur Institute, Department of Neurobiology, Laboratory of Cellular and Molecular Neurobiology-Stem Cells, Athens, Greece.

3. Institut Pasteur, Unité de Biologie des Bactéries Pathogènes à Gram-positif, CNRS UMR 2001, Paris, France

4. Hellenic Pasteur Institute, Department of Microbiology, Laboratory of Bacteriology, Athens, Greece.

5. Institut Pasteur, Ultrastructure UTechS Ultrastructural Bioimaging platform, Paris, France

\section{*Corresponding author:}

Pauline Spéder, $\mathrm{PhD}$

Department of Developmental and Stem Cell Biology

Brain plasticity in response to the environment Group

Institut Pasteur/CNRS UMR3738

25 rue du Docteur Roux

75015 PARIS

E-mail : pauline.speder@pasteur.fr

Phone: + 3314568897 


\section{$1 \quad$ Abstract}

2 Pathogens able to cross the blood-brain barrier (BBB) induce long-term neurological sequelae

3 and death. Understanding how neurotropic pathogens bypass this strong physiological barrier

4 is a prerequisite to devise therapeutic strategies. Here we propose an innovative model of

5 infection in the developing Drosophila brain, combining whole brain explants with in vivo

6 systemic infection. We identified several mammalian pathogens able to cross the Drosophila

7 BBB, including Group B Streptococcus (GBS). Amongst GBS surface components,

8 lipoproteins, and in particular the B leucin-rich Blr, were important for BBB crossing and

9 virulence in Drosophila. Further, we identified (V)LDL receptor LpR2, expressed in the BBB,

10 as a host receptor for Blr, allowing GBS translocation through endocytosis. Finally, we

11 demonstrated that Blr is required for BBB crossing and pathogenicity in a murine model of

12 infection. Our results support the relevance of Drosophila for studying host-pathogen

13 interactions and identify a new mechanism by which pathogens exploit host barriers to generate

14 brain infection. 


\section{Introduction}

2 Central nervous system (CNS) infections are rare, yet extremely damaging. They lead to fatal 3 outcomes and long-term neurological disabilities in surviving infants and adults, including 4 cognitive deficit and motor impairment ${ }^{1}$. CNS infections are caused by the entry of pathogenic agents - bacteria, fungi or viruses, from the systemic environment into the CNS, provoking neuroinflammation and cellular damages.

7 A major route for CNS infection is the bloodstream, which pathogens enter after crossing the epithelial barriers of the skin and $\mathrm{gut}^{2-4}$, and in which they circulate as free particles or carried by blood cells ${ }^{5}$. To infect the brain, pathogens must ultimately bypass an additional guardian: the blood-brain barrier $(\mathrm{BBB})^{6}$. The $\mathrm{BBB}$ is both a selective physical and chemical filter, enabling neuroprotective functions ${ }^{7}$. In higher vertebrates, brain microvascular endothelial cells form the core structure of the BBB. These cells are equipped to provide selective insulation, harboring intercellular tight junctions, absence of fenestrae, and asymmetrically localised transport systems ${ }^{8,9}$. These unique features distinguish brain microvascular endothelial cells from peripheral endothelial cells, and allow them to control ion, nutrient, and hormone transport ensuring ionic homeostasis and neuronal functions ${ }^{10,11}$. The BBB also includes perivascular pericytes, astrocytes and a basal membrane made of extracellular matrix, which regulate BBB integrity and endothelial functions ${ }^{8,12}$. This complex set of interlinked layers behaves as a double-edge sword for the organism: it restricts the entry of pathogens as well as therapeutic molecules, such as antibiotics ${ }^{13,14}$.

Pathogens that manage to cross the BBB thus secure their access to the CNS, where they tend to be immunologically-protected. Accordingly, neuro-invasive, neurotropic pathogens have developed intricate mechanisms that allow them to cross this layer and invade the $\mathrm{CNS}^{2,3,6}$. Three main strategies have been proposed so far: transcellular, paracellular and Trojan horse. The transcellular entry occurs through a receptor-mediated mechanism or pinocytosis, while the paracellular mechanism follows the increase of BBB permeability due to tight junction disruption. The Trojan horse mechanism uses infected blood cells which transmigrate from the periphery to the CNS. It has been proposed that pathogens could actually use several of these routes to invade the brain ${ }^{6}$.

30 So far, most of this knowledge comes from in vitro models of $\mathrm{BBB}^{15,16}$ where a monolayer of 31 endothelial cells are co-cultured with pericytes and astrocytes in transwells ${ }^{17}$. However, these models still display a lot of variations in their tightness depending on the endothelial cells used and thus reproducibility is a major issue. Despite induced pluripotent stem cell-related advances 
${ }^{17}$ and new set-ups like microfluidic organ-on-chips ${ }^{18}$, these models struggle to recapitulate complex parameters crucial to BBB properties, including 3D architecture and dynamic cellular interactions. Animal models, mostly mice and rats, but also zebrafish, exist and have provided essential contributions to mechanistic explorations ${ }^{3,16}$, including revisiting results from in vitro models ${ }^{19}$. Manipulating these organisms to reach cellular resolution and causal relationships is nevertheless still highly challenging. Cost and ethical issues also hinder their extensive use.

Drosophila is a powerful and tractable model system, with unrivalled genetics. It has been very successful in identifying conserved molecular mechanisms in innate immunity, such as the Toll pathway $^{20-22}$. Most studies focused on systemic and epithelial immunity (skin and gut) ${ }^{23,24}$, albeit one elegant study assessed the CNS inflammation mechanism during Zika virus infection in adults ${ }^{25}$. Strikingly, many aspects of mammalian neurogenesis are conserved in the Drosophila larva CNS, a post-embryonic, juvenile stage which also harbours a BBB (Fig. 1ab). The open circulatory system of the fly, powered by the cardiac tube, carries the hemolymph, which is in direct contact with all the organs including the CNS. The BBB represents its outermost structure. It is exclusively of glial nature, a property found in invertebrates but also in lower vertebrates, such as sharks ${ }^{26}$, and is composed of two cell layers ${ }^{27,28}$. The subperineurial glia (SPG) are large polarised cells forming an epithelium-like structure with septate junctions (Fig. 1c), the equivalent of tight junctions in vertebrates. These represent a physical barrier to paracellular diffusion, similarly to the mammalian brain vascular endothelium $^{11,29}$. The perineurial glia (PG), which do not have septate junctions, cover the SPG and are proposed to be a hemolymph sensor ${ }^{30,31}$. Transcriptional analysis of the SPG and PG layers uncovered a striking conservation of molecules with mouse BBB cells, including transporters and cell adhesion molecules ${ }^{32}$. A recent study has confirmed the relevance of the fly model, showing shared mechanisms of response to xenobiotics ${ }^{33}$. Thus, the Drosophila BBB represents a physical and chemical barrier that retains conserved chemoprotective strategies with the mammalian $\mathrm{BBB}$, ensuring brain homeostasis and protecting the brain from toxins and pathogens ${ }^{34}$.

Here we show that the Drosophila larval brain is a relevant and valuable system to model brain infection and discover cellular mechanisms of BBB crossing by mammalian pathogens. Taking advantage of this innovative model, we identified Blr, a lipoprotein displaying leucine rich repeat domains, as a new virulence factor contributing to GBS neurotropism in the fly and mouse. We further identified the Drosophila lipoprotein receptor LpR2 as a host receptor for Blr in the BBB, mediating GBS internalisation through endocytosis. 


\section{$1 \quad$ Results}

\section{Group B Streptococcus actively invades the Drosophila larval brain in an explant set up.}

3 Establishing a model of brain infection in the fly larva required developing experimental setups in which whole, intact living brains would be in contact with selected pathogens. We first devised an ex vivo protocol, as a straightforward, versatile platform for screening pathogens and conditions (Fig. 1d). Whole third instar larvae were opened posteriorly to expose all tissues while preventing damages to the brain and minimising injuries of the peripheral nerves. These brain explants, (including all larval tissues minus the gut, see Methods) were transferred to culture conditions that preserve cell viability, cell proliferation and BBB permeability, and that does not induce oxidative stress (Supp. Fig. 1a-d). In these conditions, brain explants could be kept for up to $48 \mathrm{~h}$, at $30^{\circ} \mathrm{C}$. The culture medium was then inoculated with selected pathogens at selected doses (see Methods). Brain explants were left in contact with pathogens for a given time (usually $3 \mathrm{~h}$ ) to allow binding, washed several times to remove unattached microorganisms and kept in culture until the desired time of analysis. Whole fixed brains were scanned via confocal microscopy, allowing precise localisation and quantification of individual pathogens to assess brain entry and distinguish it from adhesion (Fig. 1d).

We used this set-up to screen for prokaryotic or eukaryotic pathogens known to trigger encephalitis and/or meningitis in mammals. We found that several were able to cross the Drosophila BBB and generate brain infection $24 \mathrm{~h}$ after inoculation (Streptococcus agalactiae, Streptococcus pneumoniae, Neisseria meningitidis, Listeria monocytogenes, Candida glabrata and non-hyphal Candida albicans; Fig. 1e). In contrast, non-pathogenic strains (Lactobacillus plantarum, non-pathogenic Escherichia coli, and Saccharomyces cerevisiae) were not able to enter the Drosophila brain, pointing to specific entry mechanisms under these conditions.

Amongst the various pathogens tested, S. agalactiae (Group B Streptococcus, GBS) proved to be the most efficient to cross the fly BBB and was detected both inside the brain and attached to its surface (Fig. 2a). We thus focused on GBS.

GBS is an opportunistic gram-positive bacterium responsible for severe invasive infections in neonates developing in pneumonia, septicaemia and meningitis ${ }^{35-37}$. Despite available antibiotic treatments and intrapartum prophylaxis, these cases still represent $10 \%$ of mortality and neurological sequelae in 25 to $50 \%$ of survivors, including cognitive impairment, seizures, hearing loss, and blindness ${ }^{38,39}$. Among the various GBS clinical isolates tested, NEM316 was the most efficient to infect Drosophila larval brain explants, and chosen as our reference GBS 
strain (Fig. 2b, from now on called GBS). Interestingly, dead, formaldehyde-fixed GBS were unable to enter Drosophila brain explants, underlining the fact that GBS needs to be alive and active to cross the BBB.

\section{Dissecting the panel of strategies used by GBS to cross the multiple layers of the BBB.}

The BBB is a composite structure with several protective layers. In Drosophila, GBS has first to overcome a layer of extracellular matrix (ECM) and then the PG (Fig. 1a-b). Indeed, we found GBS embedded in a protein trap $\left(v k g: \because G F P^{40}\right)$ for collagen IV, a major, conserved ECM component of the extracellular matrix (Fig. 2c). Whereas a collagen IV layer was still present under GBS infection, it appeared disturbed, generally weaker and sometimes absent where the bacteria were detected. Interestingly, GBS displays lytic activity on a collageno-mimetic peptide (Jackson et al., 1994), suggesting that GBS could destroy the ECM to gain access to the cellular layers. The first cellular layer, the PG, does not have intercellular junctions and thus a paracellular route could be used. To determine whether GBS relies on internalisation to cross this layer, we blocked endocytosis specifically in the PG by preventing dynamin (Drosophila Shibire) function through the overexpression of a temperature-sensitive (shibire ${ }^{t s}$ ) nonfunctional form. Interestingly, we found that preventing endocytosis in this layer did not alter brain invasion (Fig. 2d). Moreover, staining for a PG membrane reporter revealed a partial and much fainter signal under infection (Fig. 2e). These alterations in PG structure were not particularly associated with GBS localisation, but rather brain-wide, suggesting a systemic origin. Interestingly, extracellular acidosis has been shown to build in the brain under meningitis ${ }^{41,42}$, and GBS is known to secrete lactic acid and acidify the culture environment ${ }^{39}$. Measuring the $\mathrm{pH}$ of the culture medium at $3 \mathrm{~h}$ post infection actually revealed a strong acidification (Supp. Fig. 2c). To assess the impact of acidification to PG disruption, we buffered the cultured medium with HEPES. Blocking medium acidification nearly completely restored PG structure (Fig. 2e). Taking these results together, we propose that disruption of the ECM precedes the bacterial traversal of the PG layer, through paracellular mechanisms or acidosisdependent destruction.

Ultimately, the capacity of GBS to invade the brain and generate infection is linked to its ability to cross the physical component of the BBB, harbouring cellular junctions ${ }^{3,37}$. This corresponds to the SPG in Drosophila. We first assayed BBB permeability of brain explants by dextran diffusion (see Methods), and found that it was significantly increased during GBS infection (Fig. 2f). We also noticed, in some cases, an altered morphology of SPG membrane and septate junctions, as assessed by the use of specific markers at confocal microscopy resolution (Supp. 
Fig. 2a-b). We assessed the contribution of acidification to these changes in BBB parameters, and found that blocking medium acidification strongly prevented the increase in $\mathrm{BBB}$ permeability observed under GBS infection (Fig. 2f), and restored BBB and septate junction morphologies (Fig. $2 \mathrm{~g}$ and Supp. Fig. 2a-b and 2d-e). Bacterial counts in the brain also significantly changed in buffered medium compared to non-buffered (Fig. 2h). However, this change was moderate, and furthermore we were able to detect bacteria attached to the brain surface through scanning electron microscopy (SEM, Supp. Fig. 2f). Altogether these data show that, although acidification might indeed help GBS by altering BBB features, it is not a prerequisite for brain entry. This argues for the critical involvement of specific mechanisms for SPG crossing by GBS, on top of acidity-induced host tissue alteration.

\section{The B Streptoccocal surface lipoprotein BIr is required for BBB crossing.}

To identify GBS surface component(s) involved in this process, we first tested known factors implicated in pathogen virulence and adhesion to endothelial cells (Fig. 3a-a'), such as the polysaccharide capsule (acapsular mutant $\Delta c p s E$ ), the hemolytic lipid toxin (non-hemolytic strain $\Delta c y l E$ and hyper-hemolytic strain $c y l+)$, or cell-wall anchored proteins $(\Delta s r t A)$. None of these mutants were strongly affecting BBB crossing (Suppl. Fig. 3a). We thus tested the contribution of lipoproteins which are tethered to the cell membrane by an N-terminal lipid moiety. In $\mathrm{Gram}^{+}$bacteria, lipoprotein biosynthesis involves two specific enzymes, i) Lgt (prolipoprotein diacylglyceryl transferase), which attaches the lipid anchor to the prolipoproteins and ii) Lsp (lipoprotein signal peptidase), which specifically removes the Nterminal signal peptide of fully mature lipoproteins. In our model, removing either Lgt or Lsp decreased bacterial count within the brain at $24 \mathrm{~h}$ post infection compared to wild-type (WT) GBS, and the double mutant ( $\Delta \operatorname{lgt} / \mathrm{l} s p$ ) displayed an additive drop (Fig. 3b), leading to a strong impairment in GBS brain entry. GBS translocation into the brain was next assessed at earlier time points and a significant decrease was also demonstrated at $6 \mathrm{~h}$ post-infection for $\Delta \operatorname{lgt} / \operatorname{lsp}$ mutant (Fig. 3c).

Next, we sought to identify specific GBS lipoprotein(s) involved in BBB crossing. The GBS repertoire consists of 39 putative GBS lipoproteins ${ }^{43}$, most of them being substrate binding proteins of ATP-binding cassette (ABC) transporters. We selected Blr (group B leucine-rich), a His-triad/Leucine-Rich Repeat (LRR) protein, as an interesting candidate (Fig. 3a'). LRR domains are classically associated with protein-protein interaction and ligand recognition ${ }^{44,45}$. Similar LRRs are actually found within the internalin $\mathrm{A}^{46}$ (InlA) of Listeria monocytogenes, a 
surface protein crucial for bacterial crossing of the gut barrier ${ }^{47}$, albeit seemingly not of the $\mathrm{BBB}^{48,49}$. To test the role of $b \operatorname{lr}$ (annotated as gbs0918), we deleted the gene in GBS NEM316.

We first checked that GBS and its isogenic mutants grew similarly in various rich laboratory media (THY, BHI) at $37^{\circ} \mathrm{C}$ as well as in Drosophila Schneider medium at $30^{\circ} \mathrm{C}$ supplemented or not with HEPES (Suppl. Fig. 3b), ruling out any effect due to growth defect. Moreover, using SEM, we found no obvious difference in morphology between WT GBS and $\Delta b l r$, that we found attached to the brain surface and in chains (Fig. 3d-e). However, as shown in Fig. 3b, $\Delta b l r$ displayed a significant decrease in bacterial count in the Drosophila larval brain at $24 \mathrm{~h}$ post infection compared to the control WT and complemented $(\Delta b l r+b l r)$ strains. GBS translocation into the brain was also significantly decreased at $6 \mathrm{~h}$ post-infection for $\Delta b l r$ mutant (Fig. 3c). Altogether, these results showed that GBS lipoproteins, and in particular Blr, are key contributors to cross the Drosophila larval BBB and enter the brain ex vivo.

Surprisingly, we noticed that infection by $\Delta b l r$ mutant resulted in significant damages to SPG membranes, altered septate junction architecture and increased BBB permeability compared to wild-type GBS (Supp. Fig. 2a-b and Supp. Fig. 3c). These differences were decreased but still remained when culture medium $\mathrm{pH}$ was maintained (Supp. Fig. 2a-b and Supp. Fig. 3c), and acidification was similar regardless of the bacterial strain (Supp. Fig. 2c), ruling out a differential effect from acidosis. Of note, none of these features were observed with the double $\Delta \operatorname{lgt} / \mathrm{lsp}$ mutant (Supp. Fig. 2a-b and Supp. Fig. 3c). In addition, using SEM, we detected the presence of additional, large structures reminiscent of the polysaccharidic coat produced during biofilm formation (Supp. Fig. 3d). Altogether, these data suggest that, in the absence of Blr, GBS turns on more destructive, yet much less efficient alternative mechanisms dependent on other lipoproteins. They also point to specific Blr-dependent mechanisms for GBS attraction to and crossing of the BBB.

\section{The Drosophila lipoprotein receptor $L p R 2$ is essential in the BBB for brain invasion by GBS.}

We then asked how the lipoprotein Blr overcomes the physical barrier of the SPG (Fig. 1a-c). Interestingly, the LRR-containing InlA was shown to interact with human E-cadherin ${ }^{50}$. We tested the role of the Drosophila E-cadherin (shotgun gene, shg) in GBS entry ex vivo, and found that specifically knocking it down in the SPG layer, through the GAL4/UAS system ${ }^{51}$, did not affect GBS brain entry (Fig. 4a). This suggests that GBS does not rely on E-cadherin for entering the fly brain. 
130 Lipoprotein receptors are conserved throughout the animal kingdom, and were originally identified as surface receptors capable of mediating cellular lipid uptake ${ }^{52}$. Lipids circulate in the blood in association with specific proteins called apolipoproteins (apo), forming lipoprotein particles of different densities (Low Density, LDL; Very Low density, VLDL). Lipoprotein receptors can be classified in two main groups, based on whether they behave as endocytic receptors supporting lipoprotein internalisation (LDLR, VLDLR, SR-A) or as mediators of lipid exchange at the cell surface. Of interest, Drosophila lipoproteins and lipoprotein receptors are similar to those in vertebrates ${ }^{53,54}$. Drosophila has seven lipoprotein receptors, belonging to the (V)LDLR families. Interestingly, specific lipoprotein particles were shown to cross the larval BBB, in which the receptors LRP1 and Megalin are expressed $30,55,56$.

140 We investigated which lipoprotein receptors were expressed in the SPG, and if they were important for GBS entry. Transcriptional data suggested expression of $l p r 1$, lpr2, arr and $m g l$ in the SPG (Pauline Spéder and Andrea H. Brand, unpublished data). Specific knockdown of

143 these receptors identified LpR2 as the main lipoprotein receptor mediating GBS entry in the 144 brain (Fig. 4a). More precisely, knocking down LpR2 in the SPG only was sufficient to decrease 145 GBS count in the brain at $24 \mathrm{~h}$ post infection. In contrast, and in accordance with our previous findings, LpR2 was not required in the PG layer for GBS brain entry (Supp. Fig. 4a). Using a gene knock-in producing an endogenous LpR2-GFP fusion (LpR2::GFP MiMIC line), we observed that LpR2 expression was restricted to the SPG, where it colocalised with a membrane marker (Fig. 4b). Similar results were obtained using an anti-LpR2 antibody (Supp. Fig. 4b-

$150 \mathrm{c}$ '). These results show that $\mathrm{LpR} 2$, a lipoprotein receptor specifically expressed in the SPG, is crucial for GBS dissemination into the brain.

GBS surface lipoprotein Blr binds to the Drosophila LpR2, allowing endocytosisdependent transcellular crossing of the BBB.

Interestingly, LpR2 has been shown to be an endocytic receptor, able to mediate the uptake of lipoprotein particles ${ }^{54,57}$. We hypothesised that binding of LpR2 to Blr could first help GBS adheres to the BBB, and ultimately lead to its internalisation into the SPG through endocytosis.

157 We first tested if LpR2 and Blr were able to physically interact. We set up a co158 immunoprecipitation experiment between the two species, incubating bacterial lysate on LpR2159 GFP fusions extracted from larval brains and bound to beads (see Methods). We showed that 160 Blr was found in the bacterial eluates from LpR2-GFP beads for wild-type and complemented 
$161(\Delta b l r+b l r)$ strains, whereas no band was recovered from $\Delta b l r$ eluates (Fig. 4c and Supp. Fig. 4d). These data showed that Drosophila LpR2 is able to bind streptococcal Blr.

163 We further assessed the role of the endocytic pathway in GBS entry. We blocked endocytosis 164 specifically in the SPG by preventing dynamin function through the overexpression of 165 temperature-sensitive (shibire $e^{t s}$ ) or dominant-negative (shibire ${ }^{D N}$ ) forms. This led to a strong 166 decrease in bacterial counts within the brain, detected as early as $6 \mathrm{~h}$ post-infection (Fig. 4d). 167 In addition, we infected larval brains in which a marker for early endosomes (Rab5-GFP) was ubiquitously expressed along a specific marker for the SPG membrane. Under these conditions we were able to detect GBS in vesicles co-staining for Rab5-GFP and SPG membranes (Fig. 4e). Expressing another early endocytic marker (FYVE-GFP) specifically in the SPG gave similar results (Supp. Fig. 4e). Finally, we were able to detect GBS in lysosomal vesicles coming from the SPG layer, through the specific expression of the Lamp1-GFP marker (Fig. 4f). Taken together, these results strongly indicate that BBB crossing by GBS occurs via endocytosis, likely through binding of Blr to LpR2 and internalisation of the resulting complexes.

\section{BIr is a virulence factor essential for BBB crossing in the Drosophila larva.}

177 This brain explant protocol in the Drosophila larva led us to uncover and propose a novel mechanism for BBB crossing by GBS, in which the pathogen surface lipoprotein Blr interacts with the host lipoprotein receptor LpR2, leading to bacterial endocytosis and CNS invasion. To confirm these findings in an in vivo set-up, we developed a protocol of brain infection through pathogen microinjection into the Drosophila circulatory system (Fig. 5a). It was preferred to feeding in order to control the dose and bypass the variability in gut crossing efficiency.

184 Bacterial counts in the brain of surviving larvae at $4 \mathrm{~h}$ and $18 \mathrm{~h}$ post injection revealed that GBS was able to access and enter the Drosophila brain via the systemic route (Fig. 5b and Supp. Fig. 5a). Survival curves ( 0 - $4 \mathrm{~h}$ post injection) showed that systemic infection by wild-type GBS increased larval lethality compared to mock injection (Fig. 5c). These results demonstrated that

188 GBS is able to infect the Drosophila brain from a circulating, systemic route, causing animal 189 mortality.

190 Next, we tested the virulence of $\Delta l g t / l s p$ and $\Delta b l r$ mutants in this set-up. First, bacterial counts

191 in the brains of surviving larvae injected with $\Delta l g t / l s p$ or $\Delta b l r$ were significantly reduced 192 compared to WT or complemented $(\Delta b l r+b l r)$ GBS strains at $4 \mathrm{~h}$ post injection (Fig. $5 \mathrm{~b})$. To 
193 discard differences in fitness or survival between these isogenic GBS strains, we determined through cfu (colony-forming units) counts (see Methods) the exact quantity of bacteria per animal: in or attached to the brain, in the hemolymph, and in all other solid tissues (Supp. Fig. $5 b)$. We then calculated three ratios: brain to hemolymph, brain to tissues, brain to hemolymph and tissues (Supp. Fig. 5c). In all cases, we found a significant decrease in $\Delta b l r$ ratios versus wild-type ratios. This shows that the loss of Blr specifically affects the neurotropic ability of GBS to adhere and/or enter the brain. In agreement with these results, survival scores were significantly higher in larvae injected with $\Delta \operatorname{lgt} / l s p$ or $\Delta b l r$ mutants compared to the two control strains, with a lethality level similar to non-infected animals (Fig. 5c).

We then assessed the role of LpR2 in the BBB during systemic infection. Infection by WT GBS of larvae in which LpR2 was specifically depleted in the SPG resulted in a dramatic reduction of bacterial count in the brain (Fig. 5d). Survival curves showed that depleting LpR2 in the SPG led to a delayed lethality compared to wild-type animals (compare black and orange curves in Fig. 5e), although it did not reach statistical significance. This suggests that, although lethality might result from brain infection, it likely also depends on a systemic effect.

\section{BIr is a virulence factor essential for BBB crossing in mice.}

209 Combining ex vivo and in vivo infection protocols allowed us to propose Blr as a virulence 210 factor of GBS essential for brain invasion in Drosophila. To determine whether this mechanism

211 is conserved in mammals, we used the mouse model of GBS hematogenous brain infection ${ }^{58}$ 212 and compared wild-type GBS strain with the isogenic $\Delta b l r$ mutant.

213 Time-course infection analysis showed that GBS could be detected in the brain as early as $3 \mathrm{~h}$ 214 post-infection, was maintained at similar levels at $6 \mathrm{~h}$ and $24 \mathrm{~h}$, and reduced at $48 \mathrm{~h}$ (Fig. 6a). 215 In parallel, bacterial counts in the blood were measurable at 3 and $6 \mathrm{~h}$ post infection and dropped 216 sharply at $24 \mathrm{~h}$ (Fig. 6b). Using a fluorescent GFP-tagged GBS, we observed bacteria attached 217 to and in the capillaries of the brain parenchyma at $4 \mathrm{~h}$ post-infection (Fig. 6c, Supp. Fig. 6a) 218 suggesting that the primary entry point for GBS is through the endothelial barrier. Interestingly, 219 we were able to detect LDLR on these capillaries, underlying the availability of this receptor at 220 GBS putative point of entry (Supp. Fig. 6b). Then, at $24 \mathrm{~h}$ after infection, we detected bacteria at the choroid plexuses and walls of the lateral (Fig. 6d), third, and fourth (not shown) ventricles,

222 that also play a barrier role in the mammalian brain. Very few cells were detected in the brain 223 parenchyma, in regions far from the ventricles, except for some small clusters in which typical 224 streptococcal chains were identified (Fig. 6d). 
225 Survival curves showed that infection with wild-type GBS led to more than $50 \%$ of lethality over 7 days (Fig. 6e). The mice that survived up to 7 days exhibited aberrant behavior indicative of neurological deficits, including unilateral palsy, immobilisation, and imbalance. Mood aberrations, such as isolation and lack of explorative behavior, were also observed. Moreover,

229 the brains of these mice revealed meningitis hallmarks including meningeal thickening and

230 leukocyte accumulation in the meninges compared with saline-injected control mice (Supp. Fig.

231 6c), as identified by co-staining for macrophages (CD68, pan-macrophage marker) and

232 microglia (Iba-1, microglia/macrophage marker).

233 In contrast, no deaths were recorded in mice infected with $\Delta b l r$ mutant and their survival curve

234 was significantly different compared to mice inoculated with WT GBS (Fig. 6e). We then 235 analysed bacterial levels in the brain and in the blood over the course of infection. The levels 236 of the $\Delta b l r$ mutant in the blood were not significantly different from wild-type GBS neither at $2373 \mathrm{~h}$ nor at $6 \mathrm{~h}$ post infection and we observed a similar clearance at $24 \mathrm{~h}$ (Supp. Fig. 6d). 238 Importantly, the brain levels of the $\Delta b l r$ mutant at $3 \mathrm{~h}$ and at $6 \mathrm{~h}$ were lower, yet not 239 significantly, and a significant reduction was then observed at $24 \mathrm{~h}$ post-infection when 240 compared with the WT strain (Supp. Fig. 6e). Normalising brain-to-blood levels confirmed that 241 the $\Delta b l r$ strain was significantly altered in its capacity to invade the mouse brain at $3 \mathrm{~h}$ and $6 \mathrm{~h}$ 242 post infection, as compared to the wild-type (Fig. 6f).

243 Interestingly, none of the mice infected with the $\Delta \operatorname{lgt} / \operatorname{lsp}$ mutant died, as observed with $\Delta b l r$ 244 mutants (Supp. Fig. 6f). Bacterial levels of $\Delta \operatorname{lgt} / \operatorname{lsp}$ mutant were reduced both in the blood and 245 brain compartments at $6 \mathrm{~h}$ post infection as compared to wild-type GBS (Supp. Fig. 6g). Yet, 246 the brain-to-blood ratios were not significantly different between these two strains (Supp. Fig. $2476 \mathrm{~h}$ ) suggesting that $\Delta \operatorname{lgt} / \mathrm{lsp}$ mutants are generally less fit in vivo.

248 Altogether, these results identify Blr as a new, conserved virulence factor endowing GBS the 249 ability to cross the BBB in Drosophila and mouse. 


\section{Discussion}

2 Here we propose an original model of brain infection, using the Drosophila larval brain, as a

3 mean to investigate molecular and cellular mechanisms contributing to the crossing of the BBB.

4 Amongst the various neurotropic pathogens tested in this model, Group B Streptococcus, a

5 pathogen responsible for meningitis in neonates and immunocompromised adults, was the most

6 efficient one and used herein. We were able to identify the surface lipoprotein Blr as a key contributor of specific GBS entry into the brain. Furthermore, we were able to show that Blr binds to LpR2, a host lipoprotein receptor expressed in the Drosophila BBB. We propose that binding of Blr to LpR2 promotes bacterial endocytosis, and GBS penetration into the brain through a transcellular passage (Fig. 7). Thus, using in vivo experiments in Drosophila, Blr and LpR2 were shown as key players in brain invasion. Blr contribution to CNS invasion was also established in a mouse model, demonstrating the power and relevance of the Drosophila model.

Our model combines an ex vivo approach with brain explants for straightforward, versatile and scalable screening of putative virulence factors and associated mechanisms, with a full in vivo approach to assess virulence and impact on the whole organism. Even though the ex vivo protocol does not allow to assess the contribution of circulating immune cells in BBB crossing, bypassing it can unveil BBB-specific mechanisms that could be masked either by an earlier, systemic effect (e.g., general inflammation) or by the difficulty to detect or assess it (e.g., acidosis). Interestingly, for example, Cryptococcus neoformans cannot enter the Drosophila larval brain in the ex vivo conditions (Fig. 1e), a finding congruent with the contribution of the Trojan horse mechanism proposed to explain C. neoformans barrier crossing ${ }^{59}$.

Adult flies have been used previously as an infection model with GBS serotype Ia (A909 strain) through pricking, unveiling the role of GBS cell-wall anchored protein alpha $\mathrm{C}$ protein and host glycosaminoglycans as important for virulence and dissemination in the adult brain ${ }^{60,61}$. We chose to investigate the larval brain in order to study the impact of infection in a developing brain, where extensive neurogenesis is happening and neural circuits are being established, mimicking more closely neonatal infections caused by GBS.

Using our model, we demonstrated the key contribution of surface-exposed lipoproteins in mediating entry of GBS into the Drosophila larval brain, and in particular the role of a specific

30 lipoprotein known as Blr. We focused our attention on Blr as an attractive candidate, because of its surface-exposed LRR region composed of 12.5 repeats of 22 amino acids. Blr was shown to be expressed in vivo but camouflaged by the bacterial capsule in GBS strain BM110. No role 
in virulence had been attributed to Blr yet. Indeed, Blr had been studied following intraperitoneal infection in a mouse model that bypasses the initial adhesion step. Interestingly though, it was shown that Blr can bind to the pathogen recognition receptor SR-A (scavenger receptor A), expressed on most macrophages and known to endocytose modified low-density lipoproteins ${ }^{62}$. This finding strongly supports our results that Blr interacts with a specific lipoprotein receptor LpR2 and is then internalised through endocytosis in the SPG. The physiological role of LpR2 in the BBB has not been described. In other tissues, LpR2, as well as the closely related LpR1, have been implicated in lipid metabolism ${ }^{54,57}$. Whole mutants for lpr 1 and $\operatorname{lpr} 2$ are actually viable, albeit with lower fertility, suggesting that these receptors are not essential at least under homeostatic conditions.

During GBS infection, some bacterial lipoproteins are released in the extracellular environment and bind Toll-like receptor 2 through their lipid moiety ${ }^{63}$. However endogenous lipoprotein receptors, which are the natural receptor for lipoproteins in vivo, have been shown to bind lipoprotein complexes through their protein component (apolipoprotein) ${ }^{64}$. In addition, it is known that lipoprotein receptors bind most of their ligands through clusters of cysteine-rich LDL receptor type-A (LA) modules. LpR2, which bears between 7 and 9 LA motifs depending on the isoform ${ }^{54}$, could thus bind Blr through its protein moiety. The LRR domain (Fig. 3a') would be of specific interest. Blr is also a virulence factor critical for BBB crossing in mice. LpR2 is orthologous to both LDLR and VLDLR proteins. Both LDLR and VLDLR have been shown to be expressed in brain endothelial cells, where they are linked to the uptake of molecular complexes across the $\mathrm{BBB}^{65-67}$. Here we confirmed LDLR localisation in blood vessels of the mouse brain in situ (Supp. Fig. 6b). Interestingly, VLDLR has been implicated in mediating the entry of Hepatitis C Virus into host cells ${ }^{68}$. The targeting of endogenous lipoprotein receptors could thus be a conserved strategy shared by a diversity of pathogens to enter cells and ultimately cross barriers, including the BBB.

Of note, we found that GBS acidifies the extracellular environment, a known parameter during meningitis. Although we demonstrated that it is not required for BBB crossing by GBS, it might help bacterial invasion, at least on a longer term, by weakening the SPG and/or affecting upstream layers, especially the PG. Indeed, lactic acid has been proposed as a virulence factor in rat fetal lung explants, where it is also linked to tissue destruction ${ }^{69}$. Interestingly, we noticed destroyed blood capillaries in the brain of mice infected with WT GBS (Supp. Fig. 6a), as well as brains with highly altered SPG during infection by WT GBS in our in vivo Drosophila model 
65 (data not shown). This suggests that acidosis-linked alteration of the BBB might be a conserved mechanism taking place during genuine infection.

67 Interestingly, Blr-deficient bacteria were less able to enter the brain while causing higher damages of the SPG. As mentioned previously, this suggests that when Blr is not available on their surface, bacteria turn to an alternative pathway, less efficient but more destructive, perhaps to gain access through alteration of junctions and tissue integrity. Such damages are not seen under infection with lipoprotein-deficient bacteria, in which GBS brain entry is extremely low.

72 This indicates first that other lipoproteins support such a pathway, and also could explain why $\Delta b l r$ still enters better than $\Delta l g t / l s p$. The presence of biofilm is intriguing, and could be a way $\Delta b l r$ causes additional damage to the BBB. Altogether, these different results underline the ability of GBS to shapeshift and use different mechanisms independently or together, depending on the conditions.

In conclusion, we propose the following model for GBS entry into the fly developing brain: rupture of the ECM, likely through GBS collagenolytic activity, and then traversal of the PG layer, through paracellular and/or destructive mechanisms. Then Blr comes at play, binds to LpR2 on the surface of the SPG allowing GBS endocytosis and brain invasion (Fig. 7). Our work, using an original model of brain infection in Drosophila, thus proposes a detailed mechanism behind pathogen crossing of the complex BBB structure, and identifies the specific

83 lipoprotein Blr as a new, conserved virulence factor for GBS. Ultimately, understanding the exact cellular and molecular pathways employed by pathogens to use and corrupt the physiological role of host receptors will provide new insight into means of helping the BBB to restrict pathogen entry during infection as well as bypassing this barrier to target therapeutics molecules. 


\section{Acknowledgments}

We thank J. Culi, C. d'Enfert, G. Janbon, F. Leulier, M-K. Taha, M. Lecuit and F. Schweisguth for reagents and strains. We are very grateful to Gunnar Lindahl for the kind gift of his homemade antibody against Blr lipoprotein. L. Arbogast generated the mdr65-mtdt-Tomato construct. Stocks obtained from the Bloomington Drosophila Stock Center (NIH P40OD018537) and from the Vienna Drosophila Resource Center were used in this study. We are grateful to A-E. Deghmane, O. Disson, C. d'Enfert, G. Janbon, M-K. Taha and M. Lecuit for their kind and enthusiastic help and advice with the pathogen screen. We are indebted to D. Ferrandon for his expert advice on the project and to S. Liégeois for technical help, especially introducing us to hemolymph injection of Drosophila larvae. We thank B. Montagne for help with the Western blots. We thank E. Voulgari for valuable advice and S. Trygoni and D. Dionysopoulou for technical support on the mouse infection model. Further, we are grateful to the personnel of the Department of Animal Models for Biomedical Research of the Hellenic Pasteur Institute for their invaluable help. We thank D. Ferrandon for critical reading of the manuscript. This work has been funded by a starting package from Institut Pasteur/ LabEx Revive and a JCJC grant from Agence Nationale de la Recherche (NeuraSteNic, ANR- 17CE13-0010-01) to P.S.; a Grand Projet Fédérateur Microbes \& Brains InFeSteR grant from Institut Pasteur to P.S., R.M. and S.D. B.B. has been supported by a Roux-Cantarini and a LabEx Revive post-doctoral fellowships.

\section{Author Contributions}

B.B. performed most Drosophila experiments. N.R. generated Fig. 2A-B, Fig. 3C, Fig. 4B-C, Supp. Fig. 4D and parts of Fig. 4D and Supp. Fig. 2A, all under the supervision of B.B. P.S. devised the brain explant protocol and started the pathogen screen. B.B. and P.S. designed and analysed all Drosophila experiments. B.P. and S.D. generated GBS strains and constructs as well as performed growth curves. S.D. designed and advised on all GBS experiments. F.P. designed, performed and analysed, together with K.S., the mouse experiments and generated Fig. 6 and Supp. Fig. 6. V.M. provided consultation on the mouse infection model and resources. R.M. supervised and advised on all mouse experiments and analysis. C.S. generated the EM and SEM data. B.B., S.D. and P.S. wrote the article with input from FP and RM.

\section{Declaration of Interests}

The authors declare no competing interest. 


\section{Figure legends}

Figure 1: Screening for mammalian neuro-invasive pathogens in a brain explant set-up identifies Group B Streptococcus as able to cross the Drosophila blood-brain barrier.

a. Schematic representation of Drosophila third instar larva showing the brain (grey) suspended in the hemolymph (orange). Top and orthogonal views of the brain covered by the blood-brain barrier (BBB) in dark orange. A'. Schematic representations of the composite Drosophila and mammalian BBBs, which include a layer of extracellular matrix (ECM in grey), a regulatory layer (perineurial glia (PG) and pericytes (PC) in light orange) and a barrier layer (subperineurial glia (SPG) and endothelial cells (EC) in dark orange) harbouring strong cell junctions (septate junctions and tight junctions in yellow). Brain cell populations, including neurons $(\mathrm{N})$ are illustrated in blue. A, anterior. P, posterior. D, dorsal. V, ventral.

b. Confocal images of the Drosophila BBB (top view and 3D orthogonal view) labelled for the ECM in grey $(v k g:: G F P)$, the PG in light orange (NP6293-GAL4>mCD8-GFP) and the SPG in red (mdr65-mtd-tomato).

c. Septate junctions in green (Lachesin::GFP).

d. Sketch of the ex vivo protocol to generate infection in Drosophila larval brains. Step 3 depicts confocal images of the brains (top and orthogonal views) stained with phalloidin (white). Pathogens (L. plantarum and S. agalactiae $[G B S]$ ) are stained in green. Orange arrows show GBS inside the brain parenchyma.

e. Screening mammalian neurotropic pathogens for their ability to cross the Drosophila BBB and invade the brain. S. agalactiae $(G B S)(\mathrm{n}=29)$, S. pneumoniae $(\mathrm{n}=14), N$. meningitidis $(\mathrm{n}$ =11), L. monocytogenes $(\mathrm{n}=43)$, and C. glabrata $(\mathrm{n}=14)$ were able to cross the Drosophila BBB. C. albicans exists in a filamentous, hyphal form linked to pathogenicity, which destroyed the BBB and brain cells $(\mathrm{n}=12)$, and in a yeast, non-hyphal form (C. albicans Afilamentous $)$ which was able to enter the brain $(\mathrm{n}=14)$. L. innocua $(\mathrm{n}=53)$ rarely crossed the $\mathrm{BBB}$, while non-pathogenic E. coli $(\mathrm{n}=12)$, L. plantarum $(\mathrm{n}=14), S$. cerevisiae and C. neoformans $(\mathrm{n}=$ 11) were not able to invade the brain.

Figure 2: GBS uses a panel of strategies to cross the multiple layers of the BBB.

a. Close-up of GBS (anti-GBS, green) attached to the SPG (mdr65-mtd-Tomato, red) as well as inside the brain. DAPI is in blue.

b. Bacterial count inside the brain $24 \mathrm{~h}$ post infection for L. plantarum $(\mathrm{n}=14), G B S B M 110$ strain (n = 29), GBS COH1 strain (n = 20), GBS NEM316 strain $(\mathrm{n}=29)$, and fixed $G B S$ NEM316 strain $(\mathrm{n}=9)$. 
c. Confocal images (top view and close up orthogonal view) of non-infected and GBS-infected brains at $6 \mathrm{~h}$ post infection showing Collagen IV staining (vkg::GFP, green) and GBS (red).

d. GBS entry at $6 \mathrm{~h}$ post infection was not significantly changed when endocytosis was blocked (dynamin $^{\text {ts }}$ ) specifically in the PG. t Student's t test: $\mathrm{p}\left(\right.$ control vs $P G>$ dynamin $\left.^{\text {ts }}\right)=0.8484$. Control (n=7); $P G>$ dynamin $^{\text {ts }}(\mathrm{n}=8)$.

e. Confocal images (top view and close up orthogonal view) of non-infected and of GBSinfected brains without and with HEPES at $6 \mathrm{~h}$ post-GBS infection, showing PG membrane (NP6293-GAL4>mCD8-GFP, light orange). GBS, green.

f. BBB permeability tests for non-infected (-) and GBS-infected brains at $6 \mathrm{~h}$ post infection without (black) and with HEPES (purple). ANOVA test: p(- vs GBS WT) <0.0001; p(- HEPES vs GBS WT HEPES $)=0.0022$; $\mathrm{p}($ GBS vs GBS+HEPES $)<0.0001$. $\mathrm{n}(-)=7$, $($ GBS WT $)=7$, $\mathrm{n}(-$ HEPES $)=8, \mathrm{n}($ GBS WT + HEPES $)=8$.

g. Transmission electron microscopy (TEM) picture of a Drosophila brain infected by GBS $6 \mathrm{~h}$ post infection, showing the different BBB layers and septate junctions (orange arrow).

h. Bacterial count inside the brain in GBS entry without (black) and with HEPES (purple) at 6 $\mathrm{h}$ (Mann-Whitney test, $\mathrm{p}=0.0102)$. $\mathrm{n}(\mathrm{GBS})=17$ and $\mathrm{n}(\mathrm{GBS}+\mathrm{HEPES})=19$.

Figure 3: Screening for surface factors identifies the lipoprotein BIr as essential for BBB crossing in Drosophila.

a-a'. Schematic representation of GBS surface structures and tested virulence factors with corresponding mutants. (A'). Schematic structure of Blr lipoprotein.

b-c. Screening of GBS surface structures and virulence factors at (b) $24 \mathrm{~h}$ and (c) $6 \mathrm{~h}$ postinfection identified GBS surface lipoproteins, and in particular Blr, as crucial for BBB crossing. Kruskal-Wallis test. (b) WT GBS $(n=31), \Delta \lg t(\mathrm{p}=0.0124, \mathrm{n}=12), \Delta l s p(\mathrm{p}=0.0022, \mathrm{n}=8)$, $\Delta \operatorname{lgt} / \operatorname{ls} p(p<0.0001, \mathrm{n}=43), \Delta b \operatorname{lr}(p<0.0001, \mathrm{n}=45), \Delta b \operatorname{lr}+\operatorname{blr}(p>0.9999, n=13)$, and $+\operatorname{blr}(p>0.9999, n=15)$. (c) WT GBS (n=19), $\Delta \operatorname{lgt} / \operatorname{ls} p(p<0.0001, \mathrm{n}=22), \Delta b \operatorname{lr}(p=0.0029$, $\mathrm{n}=16)$.

d-e. SEM picture of (d) WT GBS and (e) $\Delta b l r$ GBS attached to the brain surface.

Figure 4: Drosophila lipoprotein receptor LpR2 mediates transcellular passage of the SPG by GBS through endocytosis.

a. A knock-down screen for Drosophila E-cadherin (Shotgun) and lipoprotein receptors (LpR1, LpR2, Arrow and Megalin) identified LpR2 as crucial for BBB crossing by GBS. KruskalWallis test of each knockdown versus control: control $(\mathrm{n}=20) ; \operatorname{shg}, \mathrm{p}>0.9999(\mathrm{n}=5) ; \operatorname{lpr} 1, \mathrm{p}$ $>0.9999(\mathrm{n}=11)$; lpr2, $p=0.0003(n=18)$; arr, $\mathrm{p}>0.9999(n=5) ; m g l, \mathrm{p}>0.9999(n=6)$. 
b. Schematic representation of LpR2 structure and confocal image (top and orthogonal views) of LpR2::GFP genomic knock-in line (green) showing colocalisation of LpR2 on SPG membranes (mdr65-mtd-Tomato, red).

c. Co-immunoprecipitation experiment between LpR2::GFP immobilised on beads and bacterial lysates of WT GBS, $(\Delta b l r+b l r)$ GBS and $\Delta b l r$ GBS, detected with an antibody against Blr. A robust Blr-LpR2 interaction was revealed.

d. GBS brain invasion is endocytosis-dependent. GBS entry at $6 \mathrm{~h}$ post infection was significantly decreased by either knocking down lpr 2 or blocking endocytosis (dynamin ${ }^{\text {ts }}$ and dynamin $^{D N}$ ) specifically in the SPG. Mann-Whitney: $\mathrm{p}$ (control vs $\left.S P G>\operatorname{lpr} 2 R N A i\right)=0.0162$; $\mathrm{p}\left(\right.$ control vs $S P G>$ dynamin $\left.^{t s}\right)<0.0001 ; \mathrm{p}\left(\right.$ control vs $S P G>$ dynamin $\left.^{D N}\right)<0.0001$. Control $(\mathrm{n}=$ 25); $S P G>\operatorname{lpr} 2 R N A i(\mathrm{n}=10) ; S P G>\operatorname{dynamin}^{t s}(\mathrm{n}=12)$ and $S P G>\operatorname{dynamin}^{D N}(\mathrm{n}=7)$.

e-f. Colocalisation of GBS (white) with markers for (e) early endosome (Rab5-GFP) and (f) lysosome (Lamp1-GFP) in green within the SPG membrane (mdr65-mtd-Tomato, red).

Figure 5: An in vivo model of brain infection in Drosophila identifies Blr as a virulence factor and confirms LpR2 as a BBB receptor for brain invasion by GBS.

a. Schematic representation of Drosophila third instar larva injected with GBS. Confocal picture and close-up showing GBS (in green) inside the brain, $4 \mathrm{~h}$ after microinjection.

b. GBS brain entry 4 h post-injection for WT GBS $(\mathrm{n}=18), \Delta \operatorname{lgt} / \operatorname{sp}(\mathrm{n}=19), \Delta b l r(\mathrm{n}=23)$ and $\Delta b l r+b l r(\mathrm{n}=20)$. Kruskal Wallis test: $\Delta l g t / l s p \mathrm{p}=0.0168, \Delta b l r \mathrm{p}=0.0039, \Delta b l r+b l r \mathrm{p}=$ 0.6579 .

c. Kaplan-Meier survival curves for larvae injected with mock, WT GBS, $\Delta l g t / l s p, \Delta b l r$ and $\Delta b l r+b l r$ strains ( $\mathrm{n}=60$ for each condition) show that $\Delta l g t / l s p$ and $\Delta b l r$ are less virulent than WT GBS and $(\Delta b l r+b l r)$ GBS. Log rank test: p(GBS WT vs mock) $<0.0001$; (GBS WT vs $\Delta l g t / l s p)=0.0002 ; \mathrm{p}($ GBS WT vs $\Delta b l r)<0.0001 ; \mathrm{p}($ GBS WT vs $\Delta b l r+b l r)=0.2797$, and $\mathrm{p}($ mock vs $\Delta b l r)=0.9686$.

d. GBS brain entry at $4 \mathrm{~h}$ post injection in control $(\mathrm{n}=13)$ and $\operatorname{lpr} 2$ knockdown $(\mathrm{n}=24)$ larvae. Mann Whitney test: $\mathrm{p}<0.0001$.

e. Kaplan-Meier survival curves for control larvae and larvae in which lpr2 has been knocked down in the SPG $(S P G>\operatorname{lpr} 2 R N A i)$, injected with mock or WT GBS ( $\mathrm{n}=60$ for each condition). Log rank test: $\mathrm{p}($ Control + GBS vs $S P G>\operatorname{lpr} 2 R N A i+$ GBS $)=0.0565$; $\mathrm{p}(S P G>\operatorname{lpr} 2 R N A i$ vs $S P G>\operatorname{lpr} 2 R N A i+\mathrm{GBS})=0.0344$. 
Figure 6: Blr is a streptococcal virulence factor in mice involved in BBB crossing by GBS. a-b. GBS counts in the (a) brain $[\log 10(\mathrm{cfu} / \mathrm{g})]$ and (b) blood $[\log 10(\mathrm{cfu} / \mathrm{ml})]$ of mice inoculated with GBS WT at $3 \mathrm{~h}(\mathrm{n}=10), 6 \mathrm{~h}(\mathrm{n}=10), 24 \mathrm{~h}(\mathrm{n}=18)$, and $48 \mathrm{~h}(\mathrm{n}=10)$. Mann Whitney test: $\mathrm{p}($ Brain $24 \mathrm{~h}$ vs $48 \mathrm{~h})=0.0002$ and $\mathrm{p}($ Blood $6 \mathrm{~h}$ vs $24 \mathrm{~h})<0.0001$.

c. Confocal images showing GBS-GFP (green) attached to and in the capillaries (CD31, red) of the brain parenchyma at $4 \mathrm{~h}$ post-injection.

d. Confocal images of sagittal brain sections of mice injected with a fluorescent GBS WT-GFP strain demonstrating the presence of GFP-positive bacteria (green) at the choroid plexus (CP) inside the lateral ventricle (LV; outlined; left image) as well as at the walls of the LV and in the brain parenchyma adjacent to the LV (upper right image), at $24 \mathrm{~h}$ post infection. In the lower right image, a representative cluster of GFP-positive bacteria (also positive for anti-GBS; red) detected in the brain parenchyma. Typical streptococcal chains found in the clusters are presented in the inset. Nuclei were counterstained with DAPI and visualised in blue.

e. Kaplan-Meier survival curves of mice intravenously injected with WT GBS $(\mathrm{n}=22)$ or $\Delta b l r$ $(\mathrm{n}=10)$. Log-Rank test $\mathrm{p}=0.0055$.

f. The ratio of bacterial counts in the brain versus blood $[\log 10([\mathrm{cfu} / \mathrm{g}$ brain $] /[\mathrm{cfu} / \mathrm{ml}$ blood $])]$ in mice inoculated with $\Delta b l r$ was significantly lower than in mice inoculated with WT GBS, at 3 and $6 \mathrm{~h}$ post inoculation ( $\mathrm{n}=10$ for each condition). Student's t test, $3 \mathrm{~h}: \mathrm{p}=0.0351 ; 6 \mathrm{~h}: \mathrm{p}=$ 0.0404 .

\section{Figure 7: Proposed model for the mechanisms used by GBS during BBB crossing.}

GBS destroys the ECM layer and crosses the PG layer through a paracellular mechanism and/or cellular damages, likely supported or enhanced by acidosis. The bacteria adhere to the SPG surface via Blr-LpR2 interaction, allowing its internalisation through endocytosis and leading to its transcellular traversal. In the absence of Blr, GBS uses an alternative, albeit less efficient, mechanism for brain invasion, via SPG damages through an unknown process. 


\section{Methods}

\section{Animal models}

\section{Drosophila strains and larval culture conditions}

The following fly stocks were used: wolbachia-free $w^{1118}$ (used as reference strain for this work, ${ }^{70}$ ), $m d r 65-m t d$-tomato on (this study), mdr65-Gal4 (BDSC 50472, ${ }^{71,72}$ ), UAS-mCD8-RFP (BDSC 27399 and 27400), NP6293-Gal4 ; tub-Gal80ts (Awasaki et al., 2008), UAS-shg-RNAi (BDSC stock 34831), UAS-lpr-RNAi (VDRC stock 106364), UAS-lpr2-RNAi (VDRC stocks 107597 and 25684), UAS-arr-RNAi (VDRC stock 4818), UAS-mgl-RNAi (VDRC stock 105071),

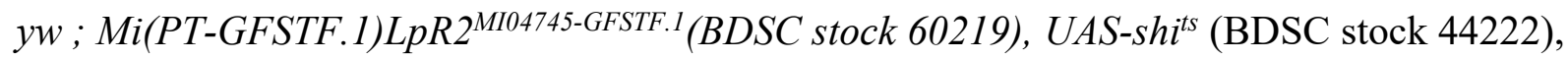
UAS-shi ${ }^{K 44 A}$ (BDSC stock 5811), yw; EGFP-Rab5 (Fabrowski et al., 2013), UAS-GFP-FYVEmyc (BDSC stock 42716), Vkg-GFP ${ }^{40}$.

Embryos were collected for 2-3 h on grape juice egg-laying plates. Equivalent numbers (100) of hatching first instar larvae were transferred to standard food plates at $25^{\circ} \mathrm{C}$ or $29^{\circ} \mathrm{C}$ (for RNAi knockdown) until mid-third instar larval stage. For the mdr65-Gal4, UAS-RFP x UAS$s h i^{t s}$, hatching first instar larvae were transferred to standard food plates at $18^{\circ} \mathrm{C}$ until earlythird instar larval stage and transferred then to $30^{\circ} \mathrm{C}$.

\section{Microorganisms used and culture conditions}

The microorganisms that were tested in our experimental set up are shown in Table 1. All strains were grown overnight at $37^{\circ} \mathrm{C}$ in BHI (Brain Heart Infusion) broth for bacteria or YPD (Yeast extract Peptone Dextrose) medium for fungi. They were stored at $-80^{\circ} \mathrm{C}$ in $\mathrm{BHI}$ broth containing $20 \%$ glycerol for bacteria or in YPD broth containing 30\% glycerol for yeast.

\section{Mouse ethics statement}

All animal experiments in this study were carried out in the Department of Animal Models for Biomedical Research of the Hellenic Pasteur Institute in strict compliance with the European and National Law for Laboratory Animals Use (Directive 2010/63/EU and Presidential Decree 156/2013), with the FELASA recommendations for euthanasia and Guide for the Care and Use of Laboratory Animals of the National Institutes of Health. All animal work was conducted according to protocols approved by the Institutional Animal Care and Use Committee of the Hellenic Pasteur Institute (Animal House Establishment Code: EL 25 BIO 013). License No 6317/27-11-2017 for experimentation was issued by the Greek authorities, i.e., the Veterinary Department of the Athens Prefecture. The preparation of this manuscript was done in compliance with ARRIVE (Animal Research: Reporting of In Vivo Experiments) guidelines. 
Table 1. Microorganisms used in this study.

\begin{tabular}{|c|c|c|}
\hline Strains & Relevant characteristics & Reference \\
\hline \multicolumn{3}{|l|}{ Bacteria } \\
\hline \multicolumn{3}{|l|}{ Escherichia coli } \\
\hline \multirow[t]{3}{*}{ DH5 $\alpha^{\mathrm{TM}}-\mathrm{pEGFP-C1}$} & $\mathrm{DH} 5 \alpha^{\mathrm{TM}}: \mathrm{F}-\Phi 80 \Delta \mathrm{l} a c Z \Delta M 15 \Delta(\operatorname{lac} Z Y A-\arg F)$ & Deghmane et al, 2011 \\
\hline & $\mathrm{U} 169$ recAl endAl hsdR $17(\mathrm{rK}-, \mathrm{mK}+)$ phoA & \\
\hline & supE44 $\lambda$ - thi-1 gyrA96 relA1 & \\
\hline \multicolumn{3}{|l|}{ Lactobacillus plantarum } \\
\hline $\mathrm{Lp}^{\mathrm{WJL}}-\mathrm{GFP}$ & & François Leulier \\
\hline Lp ${ }^{\mathrm{WJL}}$-mCherry & & François Leulier \\
\hline \multicolumn{3}{|l|}{ Streptococcus agalactiae } \\
\hline BM110 & & Lindahl et al, 2005 \\
\hline $\mathrm{COH} 1$ & & Tettelin et al, 2005 \\
\hline NEM316 & & Glaser et al 2002 \\
\hline NEM316 $\Delta c y l E$ & & Forquin et al, 2007 \\
\hline NEM316 $\Delta s r t A$ & & Lalioui et al, 2005 \\
\hline NEM316 $\Delta c p s E$ & & Chaffin et al, 2000 \\
\hline NEM316 $\Delta l g t$ & & Henneke et al, 2008 \\
\hline NEM316 $\Delta l s p$ & & Henneke et al, 2008 \\
\hline NEM316 $\Delta l g t / l s p$ & & Henneke et al, 2008 \\
\hline NEM316 $\Delta b l r$ & & This study \\
\hline $\mathrm{NEM} 316 \Delta b l r+b l r$ & & This study \\
\hline NEM316-GFP & & This study \\
\hline \multicolumn{3}{|l|}{ Streptococcus pneumoniae } \\
\hline ST4 & & Shaynoor Dramsi \\
\hline \multicolumn{3}{|l|}{ Neisseria meningitidis } \\
\hline 2C4.3-GFP & & Muhamed-kheir. Taha \\
\hline \multicolumn{3}{|l|}{ Listeria monocytogenes } \\
\hline EGDe & & Marc Lecuit \\
\hline \multicolumn{3}{|l|}{ Yeast } \\
\hline Saccharomyces cerevisiae & $\begin{array}{l}\text { MAT a his } 3 \Delta 1 \text { leu } 2 \Delta 0 \text { ura3 } \triangle 0 \text { TPll-GFP- } \\
\text { HI3Mx }\end{array}$ & Huh et al, 2003 \\
\hline \multirow[t]{3}{*}{ Candida albicans } & CEC4061 ura34- $\Delta i m m 434 / u r a 3 \Delta-\triangle i m m 434$ & Christophe D'Enfert \\
\hline & his1-hisG/his1arg44-hisG/arg4 RPS1/RPS1- & \\
\hline & Clp10-PTDH3-GFP & \\
\hline Candida glabrata & $\operatorname{trp} 1 \Delta:: \mathrm{P} T D H 3-G F P-A V A L$ & Christophe D’Enfert \\
\hline Cryptococcus neoformans & H99O-E2-Crimson & Guilhem Janbon \\
\hline
\end{tabular}




\section{Protocols}

\section{Construction of NEM316 $\Delta \mathrm{blr}$ mutant and complemented strain}

In frame deletion mutant of blr in NEM316 was constructed by using splicing-by-overlapextension PCR as previously described ${ }^{73}$. The primers used were the following:

- blr-1Eco 5'-TTCTgaattcTGTCGGTGCTGTAATGGAGT-3'

/ blr-2 5'-TAGCTCCGTAAAAGATTAGAGTCCTCCATAAATGT-3'

and

- blr-3 5'-AACATTTATGGAGGACTCTAATCTTTTACGGAGCTA-3'

/ blr-4Bam 5'-TTCTggatccAACCCCATGATGTAACACT-3'.

The chromosomal gene inactivation was carried out by cloning blr-1/blr-4 fragment into the thermosensitive shuttle plasmid $\mathrm{pG}+$ host5. Electroporation of the recombinant plasmid in $S$. agalactiae NEM316 strain and allelic exchange were performed as described ${ }^{74}$.

To complement the blr mutation in trans, the blr open reading frame was amplified using:

- pTCVblr-1Bam 5'-TCTCggatccTTATGGAGGACTCATGAAAG-3'

and

- pTCVblr-9BglII 5'- TCTCgtcgacGATTAATGGTGATGATGACC-3' primer

and cloned into the plasmid pTCV downstream from the constitutive promoter Ptet. The resulting plasmid pTCV $\Omega$ Ptet- $b l r$ was then transformed into competent NEM316 $\Delta b l r$ strain.

\section{Construction of GFP expressing NEM316}

pMV158GFP is a mobilizable plasmid harboring the $g f p$ gene cloned under the control of the $\mathrm{P}_{\mathrm{M}}$ promoter $^{75}$. pMV158GFP ${ }^{\text {Ery }}$ plasmid was constructed by replacing the Tc resistance gene of pMV158GFP by the ermB gene by using the Gibson method ${ }^{76}$. Briefly, ermB and pMV158GFP were amplified with Erm-1 5'-GAGGGTGAAATATGAACAAAAA-3' and Erm-2 5'CCCTTAACGATTTATTTCCTCC-3'primers, and pMV158-3 5'TTTTATATTTTTGTTCATATTTCACCCTCCAATAATGAGG-3' and pMV158-4 5'TATTTAACGGGAGGAAATAAATCGTTAAGGGATCAAC-3', respectively. pMV158GFP and PCR product were ligated and the resulting pMV158GFPEry was used to transform S. agalactiae NEM316 strain, applying selection for erythromycin $(10 \mu \mathrm{g} / \mathrm{ml})$.

\section{Bacterial growth curves}

One ml of overnight bacterial pre-culture in BHI was washed once in PBS and resuspended at OD600 of $2 \mathrm{ml}^{-1}$. Then each culture was diluted in a given medium at $1 / 40$ dilution and $180 \mu \mathrm{l}$ of this suspension dispensed in 96 well plates in triplicate and absorbance measurements were 
recorded using a Biotek Synergy 2 microplate reader using Gen5 data analysis software (v.3.03).

\section{DNA cloning and Drosophila transgenics}

A portion of the $m d r 65$ enhancer (GMR54C07, Flybase ID FBsf0000165529), which drives in the SPG, was amplified from genomic DNA extracted from $m d r 65-G A L 4$ adult flies, with a minimal Drosophila synthetic core promoter [DSCP ${ }^{71}$ ] fused in C-terminal. The $m t d$-Tomato DNA codes for a Tomato fluorescent protein tagged at the N-terminal end with Tag:MyrPalm (MGCCFSKT, directing myristoylation and palmitoylation) and at the C-terminal with 3 Tag:HA epitope. It was amplified from genomic DNA extracted from QUAS-mtd-Tomato adult flies (BDSC30005, Chris Potter lab). The two amplicons were joined using the Multisite gateway system ${ }^{77}$ to generate a $m d r 65^{D S C P}-m t d-$ Tomato construct. The construct was integrated in the fly genome at an attP2 docking site through PhiC31 integrase-mediated transgenesis (BestGene). Several independent transgenic lines were generated and tested, and one was kept (mdr65-mtd-Tomato).

\section{Culture of Drosophila brain explants}

Staged larvae were washed successively in PBS and ethanol 70\% v/v in water then transferred in cold Drosophila Schneider's Medium in a dissection well. Larvae were cut at around a quarter from the posterior spiracle to minimise damages to motor nerves. The posterior part was discarded and the anterior part was turned inside out to expose the brain. All larval tissues were kept except for the gut, which is removed to avoid contamination with intestinal symbiotic pathogens. Eight larvae were transferred to one well (24-well cell culture plate: Falcon 353504) and cultured in $750 \mu$ l of Culture medium I (Drosophila Schneider's medium (Gibco 21720024) supplemented with $2 \mathrm{mM}$ L-Glutamine (Gibco 25030-032) and 0.5mM Sodium L-ascorbate (Sigma A4034) at $30^{\circ} \mathrm{C}$ and $60 \%$ humidity under gentle rotary agitation (275 rpm on a Titramax 100 from Heidolph Instruments). After $3 \mathrm{~h}$, the Culture medium I is replaced by Culture medium II [Culture medium I supplemented with 1\% Fetal Bovine Serum (Sigma F4135)], then the medium was replaced after $3 \mathrm{~h}$ and every $10 \mathrm{~h}$, by a fresh Culture medium II.

\section{Drosophila brain explants infection}

An overnight preculture was set from glycerol stocks in $\mathrm{BHI}$ at $37^{\circ} \mathrm{C}$ for bacteria or in YPD at $30^{\circ} \mathrm{C}$ for yeast. The bacterial preculture was diluted $1 / 20$ in BHI, and was grown for $2 \mathrm{~h} 30 \mathrm{~min}$ at $37^{\circ} \mathrm{C}\left(\mathrm{OD}_{600}\right.$ of 0.8$)$. The yeast preculture was diluted to $\mathrm{OD}_{600}=0.2$ then grown 5 to $6 \mathrm{~h}$ at $30^{\circ} \mathrm{C}$ until $\mathrm{OD}_{600}$ of 1 . A 10x infectious dose is then prepared after pelleting through 5 minutes centrifugation at $3,500 \times \mathrm{g}\left(\right.$ at $\left.4^{\circ} \mathrm{C}\right)$, washing each original culture twice in PBS, twice in 
Drosophila Schneider's Medium and then resuspended in $750 \mu$ l of Schneider's $\left(10 \times 10^{8}\right.$ $\mathrm{CFU} / \mathrm{ml}$ for Streptococcus agalactiae, Streptococcus pneumoniae, Listeria innocua and Listeria monocytogenes; $10 \times 10^{7} \mathrm{CFU} / \mathrm{ml}$ for Neisseria meningitidis, Candida albicans and Candida glabrata and $10 \times 10^{5} \mathrm{CFU} / \mathrm{ml}$ for Cryptococcus neoformans). Pathogen concentration was calculated by $\mathrm{OD}_{600}$ correlation (Streptococcus agalactiae, Streptococcus pneumoniae, Listeria innocua and Listeria monocytogenes: $1 \mathrm{OD}_{600}=8,8 \times 10^{8} \mathrm{CFU} / \mathrm{ml}$; Neisseria meningitidis: $1 \mathrm{OD}_{600}=10^{9} \mathrm{CFU} / \mathrm{ml}$; Candida albicans and Candida glabrata: $1 \mathrm{OD}_{600}=3 \mathrm{x}$ $10^{7} \mathrm{CFU} / \mathrm{ml}$; Cryptococcus neoformans: $\left.1 \mathrm{OD}_{600}=6 \times 10^{7} \mathrm{CFU} / \mathrm{ml}\right)$.

The10 $\mathrm{x}$ infectious dose of each pathogen is diluted $1 / 10$ in the brain explant culture medium I to reach the infectious dose $\left(10^{8} \mathrm{CFU} / \mathrm{ml}\right)$. Brain explants were infected for $3 \mathrm{~h}$ at $30^{\circ} \mathrm{C}$ and $60 \%$ humidity under agitation (275 rpm on a Titramax 100 from Heidolph Instruments). Then, the infected medium was replaced by fresh culture medium II after $3 \mathrm{~h}$ and every $10 \mathrm{~h}$.

\section{Dextran Permeability}

We used $10 \mathrm{kDa}$ Dextran (Texas Red, lysine fixable, D-1863, Invitrogen) at $50 \mathrm{mM}$ final concentration in Culture medium II and we followed the same experimental and quantification procedures as described previously ${ }^{78}$.

\section{DHE assay}

To assess oxidative stress, we performed DHE (dihydroxyethidium) assay following standard procedures ${ }^{79}$. Briefly, dissected brains were incubated for $5 \mathrm{~min}$ in $30 \mu \mathrm{M}$ DHE, washed three times in PBS and then fixed for 8 minutes in 7\% formaldehyde in PBS.

\section{In vivo Drosophila larval infection}

GBS preculture and culture are prepared as described for the ex vivo protocol. $20 \mathrm{nl}$ of concentrated GBS were injected in larvae using the nano-injector Nanoject III (Drummond Scientific) in order to reach $8.8 \times 10^{8} \mathrm{CFU} / \mathrm{ml}$ of hemolymph.

\section{Immunohistochemistry}

Brains were processed and stained according to standard procedures. Briefly, brains of insideout larvae were fixed for $30 \mathrm{~min}$ in 4\% methanol-free formaldehyde (Thermo Scientific, 28908) at room temperature, washed in PBS 3 X 10 min and permeabilised in PBS-Triton $0.3 \%$ for 3 $\times 10$ min. Brains were incubated with primary antibodies at $4^{\circ} \mathrm{C}$ in blocking solution (PBSTriton 0.3\%, Bovine Serum Albumin 5\%, Normal Goat Serum 2\%) for 18-36 h, then washed with PBS-Triton $0.3 \%$ and incubated with secondary antibodies $18-24 \mathrm{~h}$ at $4^{\circ} \mathrm{C}$ in blocking solution, and washed with PBS-Triton 0.3\%, Samples were mounted in Mowiol mounting 
medium and visualised with a laser scanning confocal microscope (Zeiss LSM 880). The following primary antibodies or dyes were used: anti-GBS (homemade), anti-S.pneumoniae (homemade), anti-L.monocytogenes (R12, gift from M. Lecuit), anti-B1r ${ }^{45}$, anti-GFP (Abcam, ab13970), anti-LpR2 (gift from J. Culi), Phalloidin-Atto 647N (Sigma 65906), DAPI (Thermo 62247).

\section{Co-immunoprecipitation and western blot}

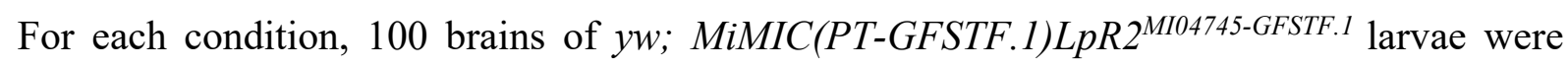
dissected and lysed in lysis buffer (50mM Tris-HCl [pH 7.5], $150 \mathrm{mM} \mathrm{NaCl,} 1 \mathrm{mM}$ DTT, noctyl-beta-glucopyranoside 1\%, 5mM EDTA, $1 \mathrm{mM}$ PMSF, protease inhibitor cocktail Roche). Brain lysates were spun for 10 minutes at $4^{\circ} \mathrm{C}$ at $15,000 \times \mathrm{g}$ and incubated $1 \mathrm{~h}$ at $4^{\circ} \mathrm{C}$ with $25 \mu \mathrm{l}$ of equilibrated agarose beads (Chromotek, bab-20) to prevent non-specific binding to beads. The brain lysates were spun for 2 mins at $4^{\circ} \mathrm{C}$ at $2,500 \times \mathrm{g}$ and the cleared supernatant was incubated overnight at $4^{\circ} \mathrm{C}$ with $25 \mu 1$ of equilibrated GFP-trap beads (Chromotek, gta-20). Bound GFP-trap beads were then washed three times, twice with lysis buffer and once with washing buffer.

Bacterial pellets of different GBS strains $W T, \Delta b l r$ and complemented $\Delta b l r+b l r$ were lysed during $1 \mathrm{~h}$ at $4^{\circ} \mathrm{C}$ with $1 \mathrm{ml}$ of lysis buffer. The bacterial lysates were spun for $10 \mathrm{~min}$ at $4^{\circ} \mathrm{C}$ at $15,000 \times \mathrm{g}$ and the supernatant was incubated for $1 \mathrm{~h}$ in $25 \mu \mathrm{l}$ of equilibrated agarose beads at $4^{\circ} \mathrm{C}$. The bacterial lysate was then spun for $2 \mathrm{~min}$ at $2,500 \times \mathrm{g}$ at $4^{\circ} \mathrm{C}$ and the cleared supernatant was incubated overnight at $4^{\circ} \mathrm{C}$ in the column containing bound GFP-trap beads. The column was spun for $2 \mathrm{~min}$ at $2,500 \times \mathrm{g}$ at $4^{\circ} \mathrm{C}$ and the beads were washed three times, once with lysis buffer and twice with washing buffer. The beads were then resuspended in Laemmli $4 \mathrm{X}$ (BioRad) with $10 \%$ of $\beta$-mercapthoethanol and heated at $90^{\circ} \mathrm{C}$ for $10 \mathrm{~min}$.

For western blot, proteins were boiled in Laemmli sample buffer, separated by SDS-PAGE on 7.5\% Mini-Protean TGX Stain-Free precast Gels (Bio-Rad, 4568024), and transferred onto PVDF membrane using the Trans-Blot Turbo transfer pack (Bio-Rad). Immuno-detection was performed as follows: the membrane was blocked in PBS-skimmed milk 5\% and incubated for 1 hour with rabbit primary anti-Blr (1/750) and mouse primary anti-GFP (Invitrogen, 1/1000, gift from B. Montagne) antibodies and then with the secondary Dylight ${ }_{680}$-coupled goat antirat antibody (Thermo Scientific Pierce) and HRP-coupled goat anti-rabbit antibody (1/10.000, gift from B. Montagne). Between the two antibodies and before detection, membranes were extensively washed with PBS $+0.1 \%$ Tween 20 and detection. was performed combining fluorescence and chemiluminescence (Bio-Rad ChemiDoc). For detection of brain lysate 
inputs, rat primary anti-GFP (1/1000, Chromotek [3H9]) and HRP-coupled goat anti rat antibody (S. Dramsi).

\section{Scanning Electron Microscopy (SEM):}

Brains were fixed overnight in 2.5\% glutaraldehyde in 0.1 M PHEM buffer $\mathrm{pH}$ 7.2. They were washed in 0.1 M PHEM buffer $\mathrm{pH} 7.2$, post-fixed for $1 \mathrm{~h}$ and $30 \mathrm{~min}$ in $1 \%$ osmium tetroxide in 0.1 M PHEM buffer pH 7.2, and then rinsed with distilled water. Samples were dehydrated through a graded series of 25, 50, 75, 95 and 100\% ethanol solutions followed by critical point drying with $\mathrm{CO} 2$.

Dried specimens were sputtered with $20 \mathrm{~nm}$ gold palladium, with a GATAN Ion Beam Coater and were examined and photographed with a JEOL JSM 6700F field emission scanning electron microscope operating at $7 \mathrm{Kv}$. Images were acquired with the upper SE detector (SEI).

\section{Transmission Electron Microscopy (TEM):}

For transmission electron microscopy, brains were fixed with $2.5 \%$ glutaraldehyde in $1 \mathrm{X}$ PHEM buffer $\mathrm{pH} 7.2$ overnight at $4^{\circ} \mathrm{C}$. Specimens were post-fixed with tannic acid $1 \%$ in $0.1 \mathrm{M}$ PHEM buffer $\mathrm{pH} 7.2$ for 30 ', post-fixed with $1 \%$ osmium tetroxide for $1 \mathrm{hr}$ and $30 \mathrm{~min}$ in $0.1 \mathrm{M}$ PHEM buffer pH7.2 at room temperature, dehydrated in a graded series of ethanol, and embedded in Epon. After heat polymerization, thin sections were cut with a Leica Ultramicrotome Ultracut UC7' sections (60 nm), stained with uranyl acetate and lead citrate. Images were taken with a Tecnai SPIRIT (FEI-Thermofisher Company at $120 \mathrm{kV}$ accelerating voltage with a camera EAGLE 4K x 4K FEI-Thermofisher Company).

\section{Mouse infection}

Eight to 10 week-old male CD-1 mice (body weight, $40.99 \pm 3.62 \mathrm{~g}$ [mean \pm standard deviation]) were randomly grouped and injected intravenously (i.v.), via the tail vein, with $10^{8}$ CFU of bacterial suspensions in sterile normal saline. For the determination of bacterial levels in blood and brain, mice were anaesthetized by intraperitoneal (i.p.) injection of a mixture containing ketamine (Imalgene 1000, MERIAL, Lyon, France; $100 \mathrm{mg} / \mathrm{kg}$ of body weight) and xylazine (Rompun, Bayer, Leverkusen, Germany; $10 \mathrm{mg} / \mathrm{kg}$ of body weight). Blood samples were collected by cardiac puncture. Immediately after, each mouse was euthanized by cervical dislocation and its brain was aseptically removed. One brain hemisphere from each mouse was homogenized in sterile normal saline. Bacterial levels in blood samples and brain homogenates were determined by plating serial tenfold dilutions on Columbia Agar with Sheep Blood plates (Thermo Fischer Scientific, Waltham, MA, USA) and counting of bacterial colonies $16 \mathrm{~h}$ later. The numbers of mice in each group of analysis are shown in Table 2. The bacterial loads per 
animal were then represented in a $\log 10$ scale, and the Brain / Blood ratios were calculated as follows: Ratio Brain/ Blood = Log10 [(cfu / g brain $) /(\mathrm{cfu} / \mathrm{ml}$ blood $)]$

Table 2. Sample size per time point per bacterial strain.

\begin{tabular}{llllll}
\multicolumn{3}{c}{ Blood and brain levels } & & Survival \\
\hline & $3 h$ & $6 h$ & $24 h$ & $48 h$ & $7 d$ \\
\hline WT GBS & 10 & 10 & 18 & 10 & 22 \\
\hline$\Delta l g t / \Delta l s p$ & 12 & 17 & 10 & 10 \\
\hline$\Delta b l r$ & 10 & 10 & 9 & & 10 \\
\hline
\end{tabular}

\section{Mouse immunohistology}

Mice were euthanized by (i.p.) injection of a ketamine/xylazine mix. After transcardial perfusion with $4 \%$ paraformaldehyde in phosphate-buffered saline (PBS), the brains of the infected mice were dissected out, post-fixed in the same fixative, cryoprotected in $30 \% \mathrm{w} / \mathrm{v}$ sucrose solution in PBS for $2 \mathrm{~d}$ at $4{ }^{\circ} \mathrm{C}$, embedded in O.C.T. compound (VWR Chemicals) and frozen at $-80^{\circ} \mathrm{C}$. Series of coronal or sagittal $20 \mu \mathrm{m}$-thick sections were collected on Superfrost Plus microscope slides and stored at $-20{ }^{\circ} \mathrm{C}$ until further processing. The cryosections were thawed and subjected to antigen retrieval in $10 \mathrm{mM}$ sodium citrate solution, $\mathrm{pH} 6$, followed by $1 \mathrm{~h}$ blocking of non-specific sites with $5 \% \mathrm{v} / \mathrm{v}$ normal donkey serum (NDS), simultaneously with permeabilization using $0.1 \% \mathrm{v} / \mathrm{v}$ Triton X-100 in PBS. Primary antibodies diluted in $2.5 \%$ NDS in PBS were applied overnight at $4{ }^{\circ} \mathrm{C}$, followed by incubation with the appropriate secondary antibodies for $2 \mathrm{~h}$ at room temperature. The following primary antibodies were used: rat anti-Cluster of Differentiation 68 (CD68; 1:100; Bio-Rad Antibodies, Oxford, UK; MCA1957GA), rabbit polyclonal anti-ionized calcium-binding adapter molecule 1 (Iba-1; 1:400; FUJIFILM Wako Pure Chemical Corporation, Osaka, Japan; 019-19741), rabbit antiCD31(1:50; Abcam, Cambridge, UK; ab28364), goat anti-LDLR (1:100, R\&D Systems, MN, USA; AF2255), rabbit anti-GBS (1:300; home-made). Secondary antibodies (all from Thermo Fisher Scientific) used for immunofluorescence were conjugated with Alexa Fluor 488 or 546 and cell nuclei were counterstained with 4',6-diamidino-2-phenylindole (DAPI; 1:1000; Thermo Fisher Scientific). Prolong Gold antifade curing mountant (Cell Signaling Technology, Danvers, MA, USA) was used for mounting. Images were acquired using Leica TCS SP8 confocal microscope. 


\section{Image processing}

Fiji, Icy or Volocity were used to process confocal data. Adobe Photoshop and Illustrator were used to assemble figures.

\section{Statistics}

GraphPad Prism software was used for all analyses.

\section{Bacterial quantifications in infected Drosophila brain}

The same region of the CNS (Ventral Nerve Cord,VNC) was scanned at an optimised number of slices using a Zeiss LSM880 microscope. The exact number of bacteria for each brain was then determined manually by counting each individual bacterium contained within the boundary of the BBB (mdr65-mtd-Tomato).

\section{CFU counts following in vivo larval GBS-injection}

Each injected larva is washed on a paper with ethanol 70\% then bled in $10 \mu 1$ PBS. The brain is then dissected, transferred and homogenized in $10 \mu \mathrm{PBS}$. The rest of the larval carcass (other tissues except the gut) is also transferred and homogenized in $10 \mu 1$ PBS. This protocol was done for 5 larvae by condition. Hemolymph, brain and carcass bacterial levels were determined by plating 7 serial tenfold dilutions two times on Columbia Agar with Sheep Blood plates (Biomérieux 43041) and counting of bacterial colonies after 16 hours at $37^{\circ} \mathrm{C}$. The average $\mathrm{CFU} / \mu \mathrm{l}$ was calculated as an average from all the different dilutions. The bacterial loads per animal were then represented in a Log10 scale, and ratios were calculated from raw counting then represented in a $\log 10$ scale:

- Ratio Brain / Hemolymph = Log10 (cfu per brain / cfu per hemolymph)

- Ratio Brain / Other tissues = Log10 (cfu per brain / cfu per Other tissues)

- Ratio Brain / (Hemolymph+Other tisses) = Log10 (cfu per brain / (cfu per hemolymph+ cfu per Other tissues))

\section{Drosophila statistical analysis:}

- In order to perform statistical tests on several experimental replicates, each value (corresponding to one brain) was normalised to the mean of the control condition within one replicate. Statistical tests were then run on all the normalised values from all replicates, which were considered as biological replicates.

- Comparisons between BBB permeability, GBS entry into the brain, cell viability, oxidative stress, bacterial levels in the hemolymph, bacterial levels in other tissues, bacterial levels in the brain, ratio of bacterial levels for brain/hemolymph, ratio of bacterial levels for brain/other 
tissues and ratio of bacterial levels for brain/other tissues+hemolymph were performed by Student's t test ( 2 conditions) or one-way ANOVA test followed by Tukey's post-hoc analysis (more than 2 conditions) when values followed a normal distribution (assessed by ShapiroWilk normality test). Otherwise, non-parametric Mann-Whitney tests ( 2 conditions) or KruskalWallis tests (more than 2 conditions) were performed.

- Comparison of survival curves was performed using Log-rank test.

- P values lower than 0.05 were considered significant.

\section{Mouse statistical analysis:}

- Comparisons between bacterial levels in the blood and the brain, as well as between ratios of bacterial levels for brain/blood were performed by unpaired Student's t test when values followed a normal distribution (assessed by D'Agostino-Pearson normality test). Otherwise, non-parametric Mann-Whitney test was performed.

- Comparison of survival curves was performed using Log-rank test.

- P values lower than 0.05 were considered significant. 


\section{References}

1. John, C. C. et al. Global research priorities for infections that affect the nervous system. Nature 527, S178-S186 (2015).

2. Cain, M. D., Salimi, H., Diamond, M. S. \& Klein, R. S. Mechanisms of Pathogen Invasion into the Central Nervous System. Neuron 103, 771-783 (2019).

3. Dando, S. J. et al. Pathogens penetrating the central nervous system: infection pathways and the cellular and molecular mechanisms of invasion. Clin. Microbiol. Rev. 27, 691-726 (2014).

4. Doran, K. S., Banerjee, A., Disson, O. \& Lecuit, M. Concepts and mechanisms: Crossing host barriers. Cold Spring Harb. Perspect. Med. 3, (2013).

5. Kristensson, K. Microbes' roadmap to neurons. Nature Reviews Neuroscience 12, 345357 (2011).

6. Coureuil, M., Lécuyer, H., Bourdoulous, S. \& Nassif, X. A journey into the brain: insight into how bacterial pathogens cross blood-brain barriers. Nat. Rev. Microbiol. 15, 149-159 (2017).

7. Daneman, R. \& Prat, A. The Blood - Brain Barrier. 1-24 (2015). doi:10.1002/ana.23648

8. Siegenthaler, J. a, Sohet, F. \& Daneman, R. 'Sealing off the CNS': cellular and molecular regulation of blood-brain barriergenesis. Curr. Opin. Neurobiol. 1-8 (2013).

doi:10.1016/j.conb.2013.06.006

9. Saunders, N. R., Habgood, M. D., Møllgård, K. \& Dziegielewska, K. M. The biological significance of brain barrier mechanisms: help or hindrance in drug delivery to the central nervous system? F1000Research 5, (2016).

10. Lampron, A., ElAli, A. \& Rivest, S. Innate Immunity in the CNS: Redefining the relationship between the CNS and its Environment. Neuron 78, 214-232 (2013).

11. Weiler, A., Volkenhoff, A., Hertenstein, H. \& Schirmeier, S. Metabolite transport across the mammalian and insect brain diffusion barriers. Neurobiol. Dis. 107, 15-31 (2017).

12. Obermeier, B., Daneman, R. \& Ransohoff, R. M. Development, maintenance and disruption of the blood-brain barrier. Nat. Med. 19, 1584-96 (2013).

13. Banks, W. a. Brain meets body: the blood-brain barrier as an endocrine interface. Endocrinology 153, 4111-9 (2012).

14. Daneman, R. The blood-brain barrier in health and disease. Ann. Neurol. 72, 648-72 (2012). 
15. Sivandzade, F. \& Cucullo, L. In-vitro blood-brain barrier modeling: A review of modern and fast-advancing technologies. J. Cereb. Blood Flow Metab. 38, 1667-1681 (2018).

16. Jackson, S. et al. Model systems for studying the blood-brain barrier: Applications and challenges. Biomaterials 214, 119217 (2019).

17. Bergmann, S. et al. Blood-brain-barrier organoids for investigating the permeability of CNS therapeutics. Nat. Protoc. 13, 2827-2843 (2018).

18. Heidari, H. \& Taylor, H. Review Article: Capturing the physiological complexity of the brain's neuro-vascular unit in vitro. Biomicrofluidics 12, 051502 (2018).

19. Disson, O. \& Lecuit, M. In vitro and in vivo models to study human listeriosis: mind the gap. Microbes Infect. 15, 971-80 (2013).

20. Lemaitre, B. \& Hoffmann, J. The Host Defense of Drosophila melanogaster. Annu. Rev. Immunol. 25, 697-743 (2007).

21. Bier, E. \& Guichard, A. Deconstructing host-pathogen interactions in Drosophila. Dis. Model. Mech. 5, 48-61 (2012).

22. Péan, C. B. \& Dionne, M. S. Intracellular infections in Drosophila melanogaster: host defense and mechanisms of pathogenesis. Dev. Comp. Immunol. 42, 57-66 (2014).

23. Ferrandon, D. The complementary facets of epithelial host defenses in the genetic model organism Drosophila melanogaster: from resistance to resilience. Curr. Opin. Immunol. 25, 59-70 (2013).

24. Bergman, P., Seyedoleslami Esfahani, S. \& Engström, Y. Drosophila as a Model for Human Diseases-Focus on Innate Immunity in Barrier Epithelia. Curr. Top. Dev. Biol. 121, 29-81 (2017).

25. Liu, Y. et al. Inflammation-Induced, STING-Dependent Autophagy Restricts Zika Virus Infection in the Drosophila Brain. Cell Host Microbe 24, 57-68.e3 (2018).

26. Bundgaard, M. \& Abbott, N. J. All vertebrates started out with a glial blood-brain barrier 4-500 million years ago. Glia 56, 699-708 (2008).

27. Stork, T. et al. Organization and function of the blood-brain barrier in Drosophila. $J$. Neurosci. 28, 587-97 (2008).

28. Limmer, S., Weiler, A., Volkenhoff, A., Babatz, F. \& Klämbt, C. The Drosophila bloodbrain barrier: development and function of a glial endothelium. Front. Neurosci. 8, 365 (2014).

29. Hindle, S. J. \& Bainton, R. J. Barrier mechanisms in the Drosophila blood-brain barrier. 
Front. Neurosci. 8, 414 (2014).

30. DeSalvo, M. K., Mayer, N., Mayer, F. \& Bainton, R. J. Physiologic and anatomic characterization of the brain surface glia barrier of Drosophila. Glia 59, 1322-40 (2011).

31. Parkhurst, S. J. et al. Perineurial Barrier Glia Physically Respond to Alcohol in an Akap200-Dependent Manner to Promote Tolerance. Cell Rep. 22, 1647-1656 (2018).

32. DeSalvo, M. K. et al. The Drosophila surface glia transcriptome: evolutionary conserved blood-brain barrier processes. Front. Neurosci. 8, 346 (2014).

33. Hindle, S. J. et al. Evolutionarily Conserved Roles for Blood-Brain Barrier Xenobiotic Transporters in Endogenous Steroid Partitioning and Behavior. Cell Rep. 21, 1304-1316 (2017).

34. Mayer, F. et al. Evolutionary conservation of vertebrate blood-brain barrier chemoprotective mechanisms in Drosophila. J. Neurosci. 29, 3538-50 (2009).

35. Doran, K. S. \& Nizet, V. Molecular pathogenesis of neonatal group B streptococcal infection: no longer in its infancy. Mol. Microbiol. 54, 23-31 (2004).

36. Rajagopal, L. Understanding the regulation of Group B Streptococcal virulence factors. Future Microbiol. 4, 201-221 (2009).

37. Shabayek, S. \& Spellerberg, B. Group B Streptococcal Colonization, Molecular Characteristics, and Epidemiology. Front. Microbiol. 9, 437 (2018).

38. Schuchat, A. Group B streptococcus. Lancet 353, 51-56 (1999).

39. Libster, R. et al. Long-term Outcomes of Group B Streptococcal Meningitis. Pediatrics 130, e8-e15 (2012).

40. Buszczak, M. et al. The carnegie protein trap library: a versatile tool for Drosophila developmental studies. Genetics 175, 1505-31 (2007).

41. Andersen, N. E. O., Gyring, J., Hansen, A. J., Laursen, H. \& Siesjö, B. K. Brain Acidosis in Experimental Pneumococcal Meningitis. J. Cereb. Blood Flow Metab. 9, 381-387 (1989).

42. Eross, J., Silink, M. \& Dorman, D. Cerebrospinal fluid lactic acidosis in bacterial meningitis. Arch. Dis. Child. 56, 692-698 (1981).

43. C. Sutcliffe, I. \& J. Harrington, D. Putative lipoproteins of Streptococcus agalactiae identified by bioinformatic genome analysis. Antonie Van Leeuwenhoek 85, 305-315 (2004). 44. Kobe, B. \& Kajava, A. V. The leucine-rich repeat as a protein recognition motif. Curr. Opin. Struct. Biol. 11, 725-32 (2001). 
45. Waldemarsson, J., Areschoug, T., Lindahl, G. \& Johnsson, E. The streptococcal Blr and Slr proteins define a family of surface proteins with leucine-rich repeats: camouflaging by other surface structures. J. Bacteriol. 188, 378-88 (2006).

46. Lecuit, M., Ohayon, H., Braun, L., Mengaud, J. \& Cossart, P. Internalin of Listeria monocytogenes with an intact leucine-rich repeat region is sufficient to promote internalization. Infect. Immun. 65, 5309-19 (1997).

47. Pizarro-Cerdá, J., Kühbacher, A. \& Cossart, P. Entry of listeria monocytogenes in mammalian epithelial cells: An updated view. Cold Spring Harb. Perspect. Med. 2, a010009 (2012).

48. Bergmann, S. et al. Influence of internalin a murinisation on host resistance to orally acquired listeriosis in mice. BMC Microbiol. 13, 90 (2013).

49. Ghosh, P. et al. Invasion of the brain by Listeria monocytogenes is mediated by InlF and host cell vimentin. MBio 9, (2018).

50. Mengaud, J., Ohayon, H., Gounon, P., Mege, R. M. \& Cossart, P. E-cadherin is the receptor for internalin, a surface protein required for entry of $L$. monocytogenes into epithelial cells. Cell 84, 923-932 (1996).

51. Brand, A. H. \& Perrimon, N. Targeted gene expression as a means of altering cell fates and generating dominant phenotypes. Development 118, 401-15 (1993).

52. Dieckmann, M., Dietrich, M. F. \& Herz, J. Lipoprotein receptors--an evolutionarily ancient multifunctional receptor family. Biol. Chem. 391, 1341-63 (2010).

53. Palm, W. et al. Lipoproteins in Drosophila melanogaster--assembly, function, and influence on tissue lipid composition. PLoS Genet. 8, e1002828 (2012).

54. Parra-Peralbo, E. \& Culi, J. Drosophila lipophorin receptors mediate the uptake of neutral lipids in oocytes and imaginal disc cells by an endocytosis-independent mechanism. PLoS Genet. 7, (2011).

55. Brankatschk, M. \& Eaton, S. Lipoprotein particles cross the blood-brain barrier in Drosophila. J. Neurosci. 30, 10441-7 (2010).

56. Brankatschk, M., Dunst, S., Nemetschke, L. \& Eaton, S. Delivery of circulating lipoproteins to specific neurons in the Drosophila brain regulates systemic insulin signaling. Elife 3, 1-19 (2014).

57. Rodríguez-Vázquez, M., Vaquero, D., Parra-Peralbo, E., Mejía-Morales, J. E. \& Culi, J. Drosophila Lipophorin Receptors Recruit the Lipoprotein LTP to the Plasma Membrane to 
Mediate Lipid Uptake. PLOS Genet. 11, e1005356 (2015).

58. Banerjee, A. et al. Bacterial Pili exploit integrin machinery to promote immune activation and efficient blood-brain barrier penetration. Nat. Commun. 2, 462 (2011).

59. Santiago-Tirado, F. H., Onken, M. D., Cooper, J. A., Klein, R. S. \& Doering, T. L. Trojan Horse Transit Contributes to Blood-Brain Barrier Crossing of a Eukaryotic Pathogen. MBio 8, (2017).

60. Baron, M. J., Wong, S. L., Nybakken, K., Carey, V. J. \& Madoff, L. C. Host Glycosaminoglycan Confers Susceptibility to Bacterial Infection in Drosophila melanogaster. Infect. Immun. 77, 860-866 (2008).

61. Chang, Y.-C. et al. Glycosaminoglycan binding facilitates entry of a bacterial pathogen into central nervous systems. PLoS Pathog. 7, e1002082 (2011).

62. Areschoug, T., Waldemarsson, J. \& Gordon, S. Evasion of macrophage scavenger receptor A-mediated recognition by pathogenic streptococci. Eur. J. Immunol. 38, 3068-3079 (2008).

63. Henneke, P. et al. Lipoproteins are critical TLR2 activating toxins in group B streptococcal sepsis. J. Immunol. 180, 6149-58 (2008).

64. Blacklow, S. C. Versatility in ligand recognition by LDL receptor family proteins: advances and frontiers. Curr. Opin. Struct. Biol. 17, 419-26 (2007).

65. Pan, W. et al. Efficient transfer of receptor-associated protein (RAP) across the bloodbrain barrier. J. Cell Sci. 117, 5071-8 (2004).

66. Deane, R. et al. apoE isoform-specific disruption of amyloid beta peptide clearance from mouse brain. J. Clin. Invest. 118, 4002-13 (2008).

67. Molino, Y. et al. Use of LDL receptor-targeting peptide vectors for in vitro and in vivo cargo transport across the blood-brain barrier. FASEB J. 31, 1807-1827 (2017).

68. Ujino, S. et al. Hepatitis C virus utilizes VLDLR as a novel entry pathway. Proc. Natl. Acad. Sci. U. S. A. 113, 188-93 (2016).

69. Kling, D. E. et al. Lactic acid is a potential virulence factor for group B Streptococcus. Microb. Pathog. 46, 43-52 (2009).

70. Pontier, S. M. \& Schweisguth, F. A Wolbachia-Sensitive Communication between Male and Female Pupae Controls Gamete Compatibility in Drosophila. Curr. Biol. 25, 2339-48 (2015).

71. Pfeiffer, B. D. et al. Tools for neuroanatomy and neurogenetics in Drosophila. Proc. 
Natl. Acad. Sci. U. S. A. 105, 9715-20 (2008).

72. Spéder, P. \& Brand, A. H. Gap Junction Proteins in the Blood-Brain Barrier Control Nutrient-Dependent Reactivation of Drosophila Neural Stem Cells. Dev. Cell 309-321 (2014). doi:10.1016/j.devcel.2014.05.021

73. Dramsi, S. et al. Assembly and role of pili in group B streptococci. Mol. Microbiol. 60, 1401-1413 (2006).

74. Biswas, I., Gruss, A., Ehrlich, S. D. \& Maguin, E. High-efficiency gene inactivation and replacement system for gram-positive bacteria. J. Bacteriol. 175, 3628-35 (1993).

75. Nieto, C. \& Espinosa, M. Construction of the mobilizable plasmid pMV158GFP, a derivative of pMV158 that carries the gene encoding the green fluorescent protein. Plasmid 49, 281-5 (2003).

76. Gibson, D. G. et al. Enzymatic assembly of DNA molecules up to several hundred kilobases. Nat. Methods 6, 343-345 (2009).

77. Petersen, L. K. \& Stowers, R. S. A Gateway MultiSite recombination cloning toolkit. PLoS One 6, e24531 (2011).

78. Hatan, M., Shinder, V., Israeli, D., Schnorrer, F. \& Volk, T. The Drosophila blood brain barrier is maintained by GPCR-dependent dynamic actin structures. J. Cell Biol. 192, 307-19 (2011).

79. Owusu-Ansah, E., Yavari, A. \& Banerjee, U. A protocol for in vivo detection of reactive oxygen species. Protoc. Exch. (2008). doi:10.1038/nprot.2008.23 
Figure 2

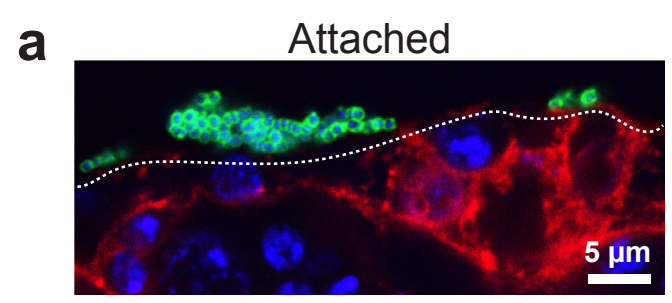

Inside

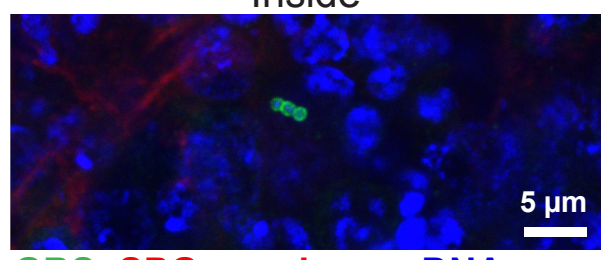

GBS SPG membrane DNA

C

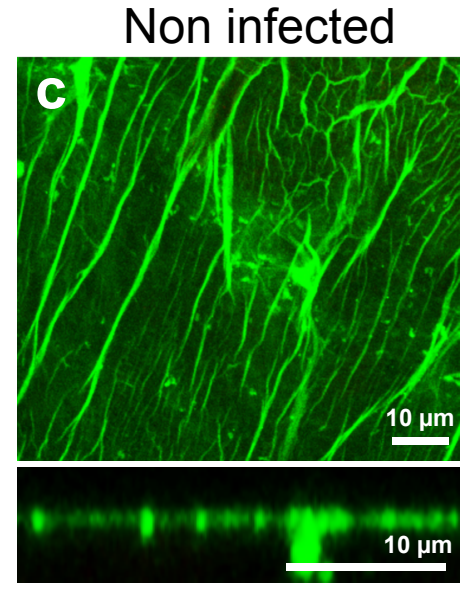

GBS

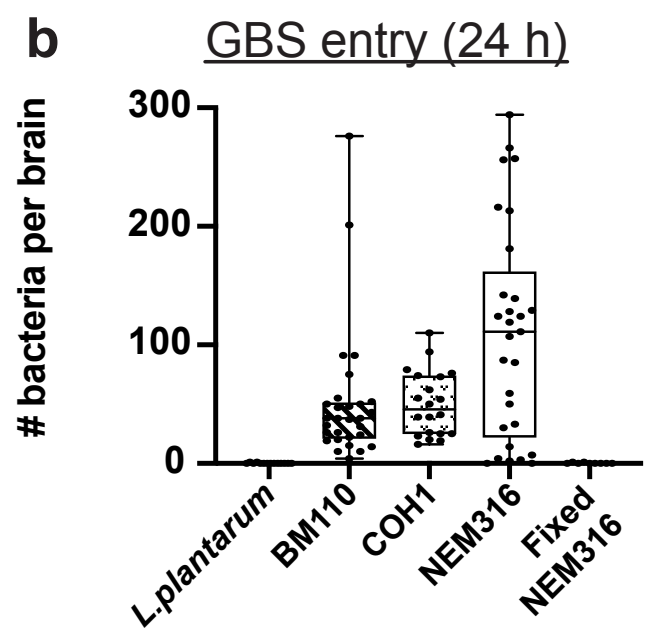

GBS $6 \mathrm{~h}$

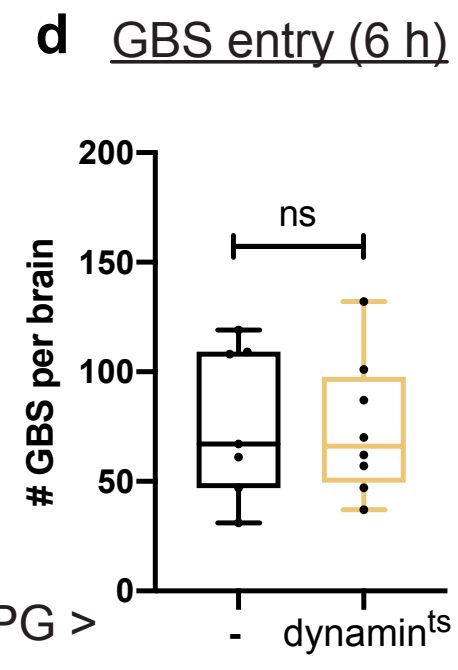

f Permeability (6h)

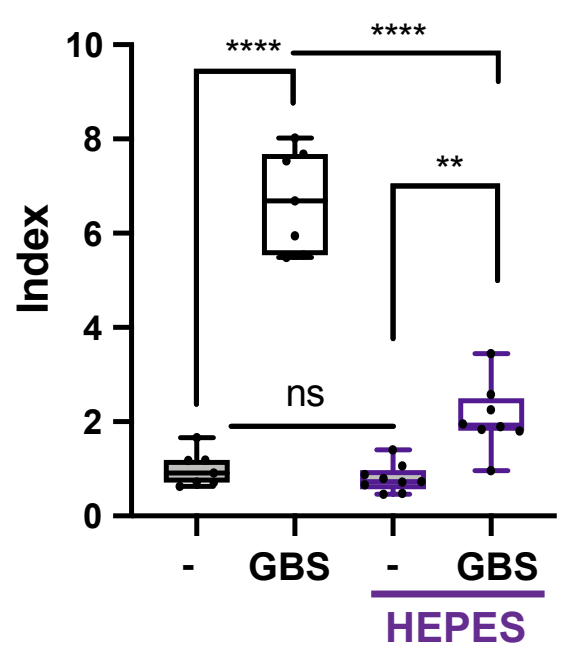

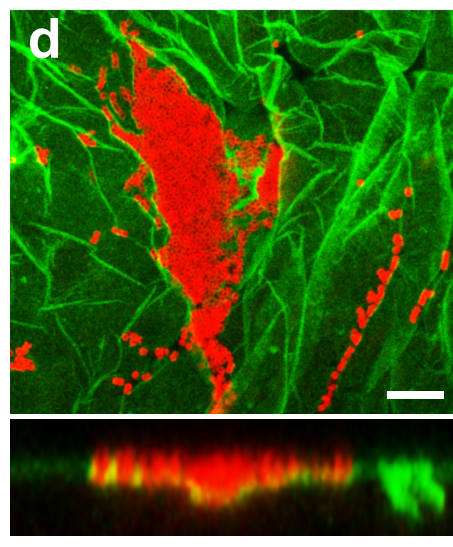

CollV::GFP GBS
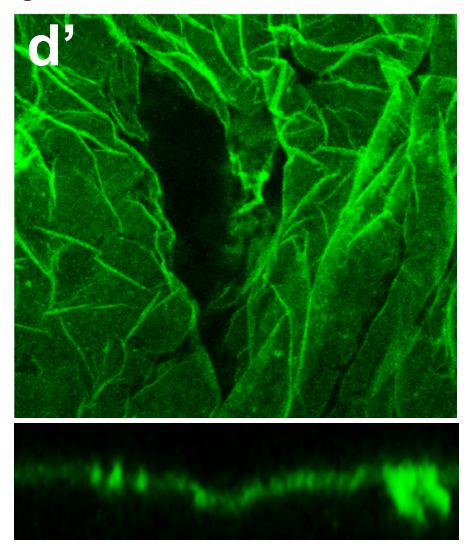

CollV:: GFP

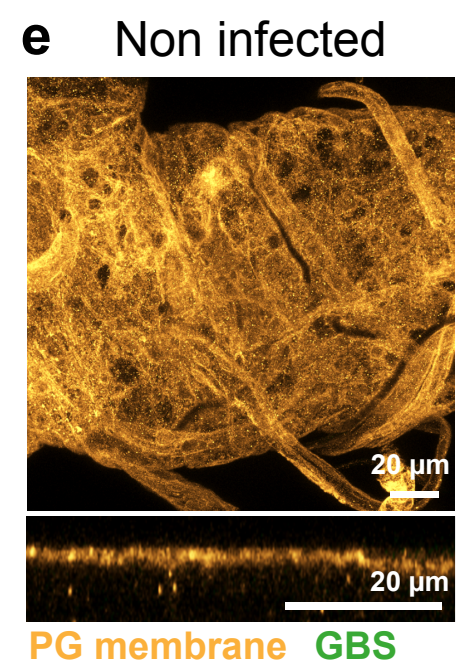

GBS $6 \mathrm{~h}$

GBS $6 \mathrm{~h}+$ HEPES
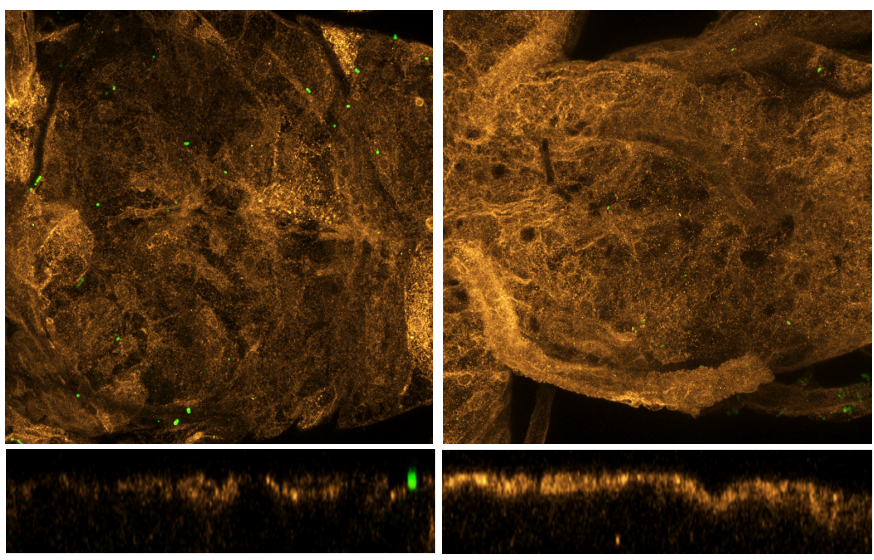

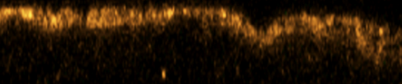


Figure 3

a

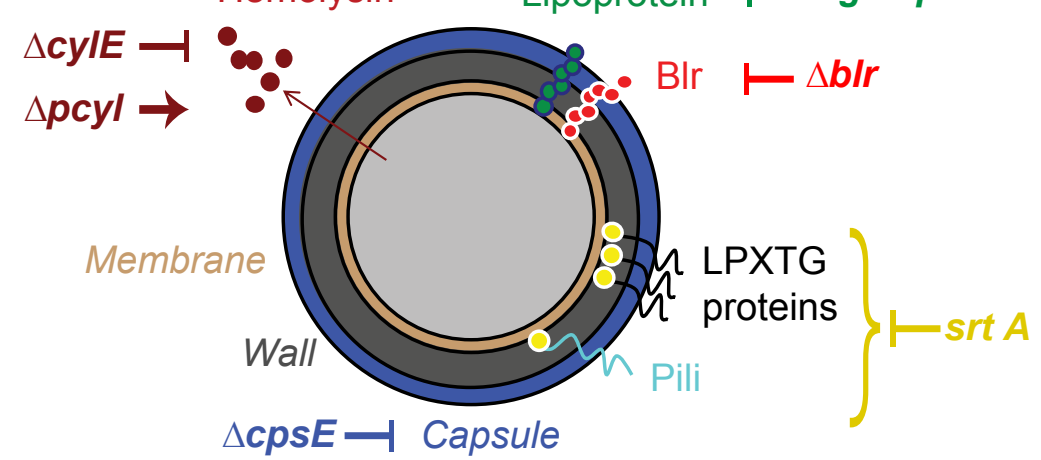

$a^{\prime}$

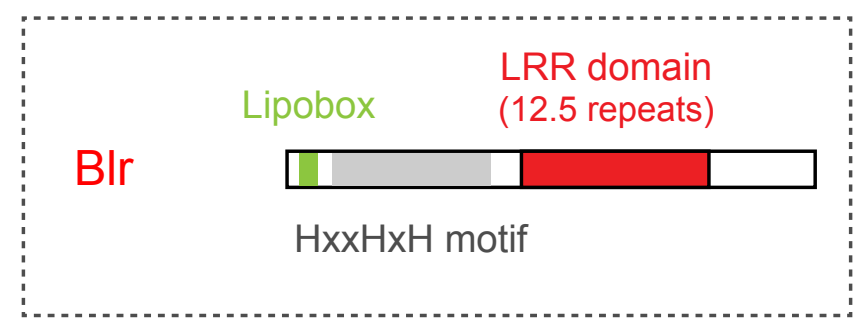

GBS entry in the brain

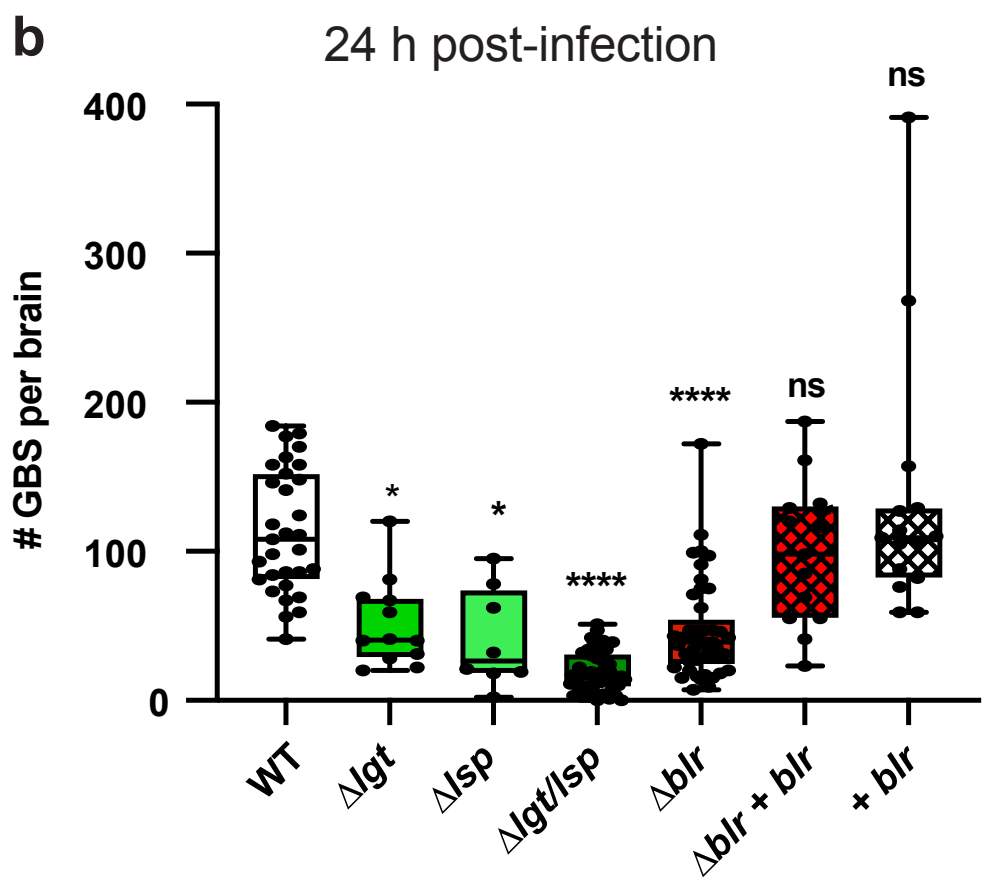

C $6 \mathrm{~h}$ post-infection

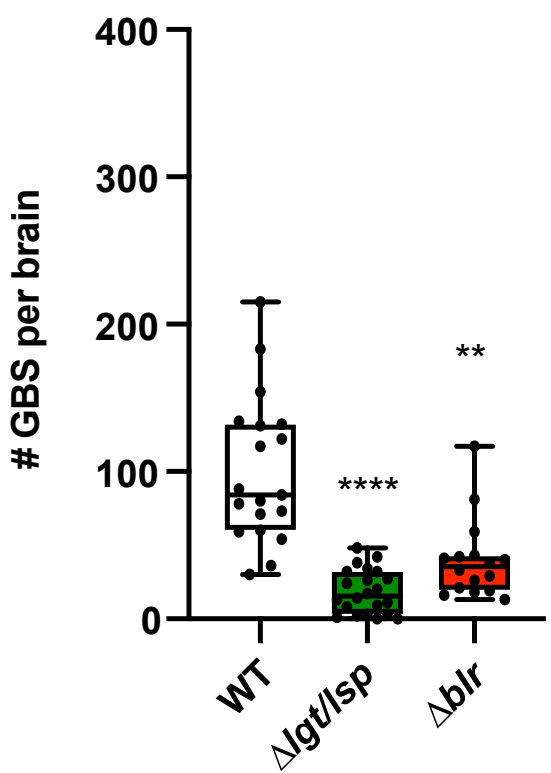

WT GBS $6 \mathrm{~h}$ + HEPES

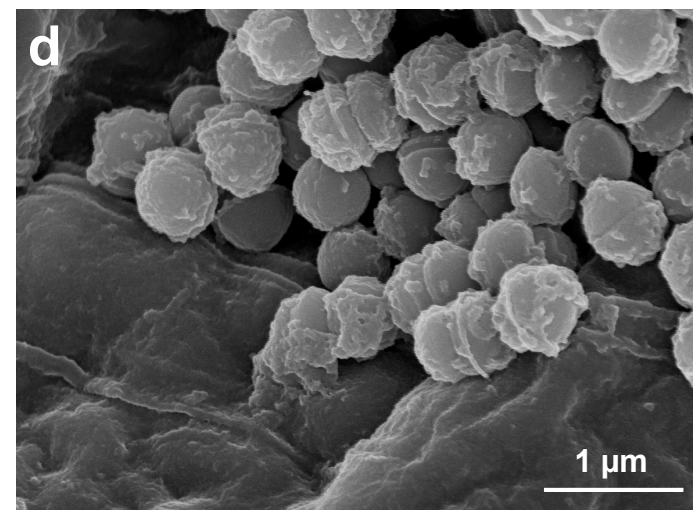

$\Delta b / r 6 \mathrm{~h}+$ HEPES

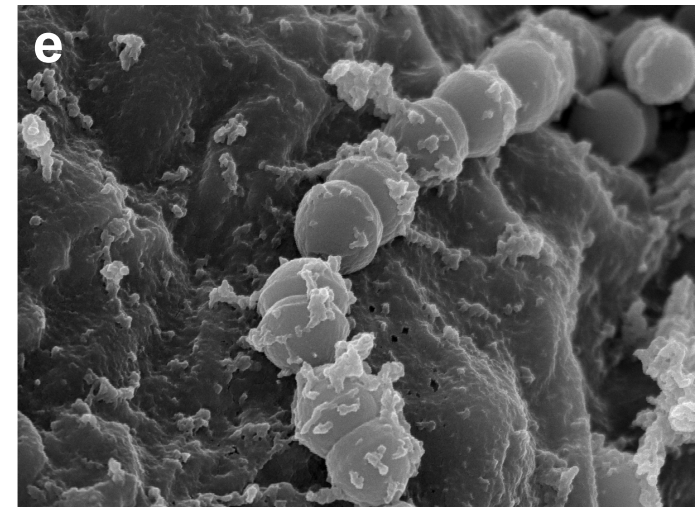


Figure 4

a

GBS entry (24 h)

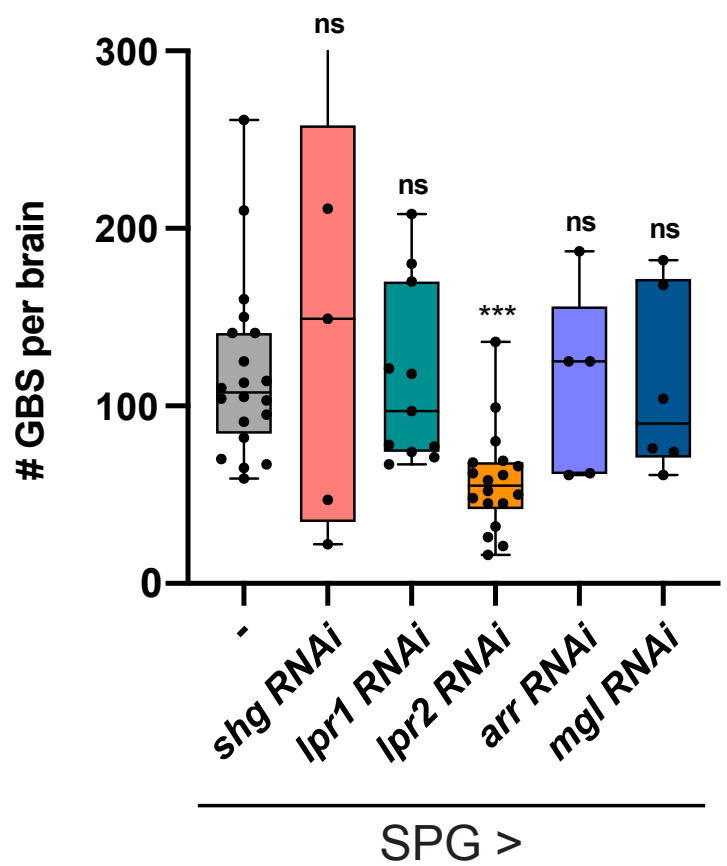

b

Transmembrane

EGF domain

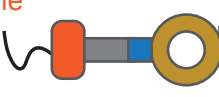

$\beta$-propeller

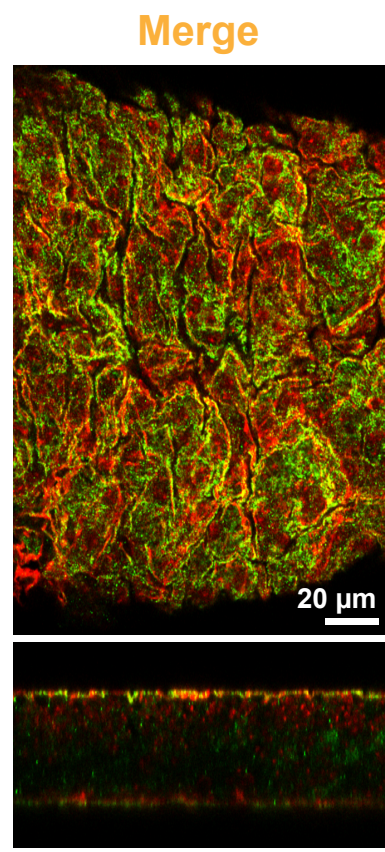

SPG membrane
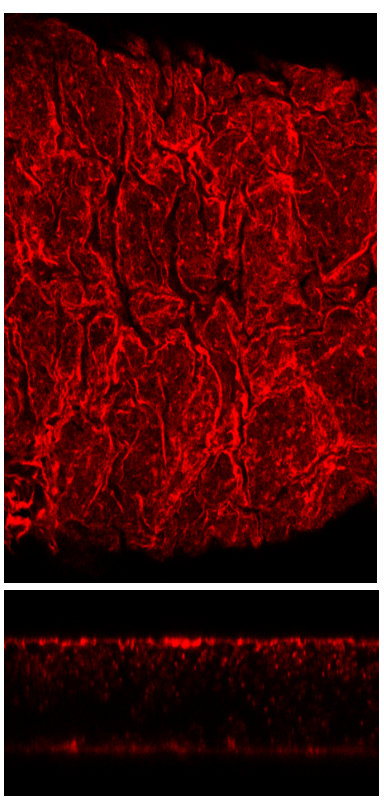

d
C
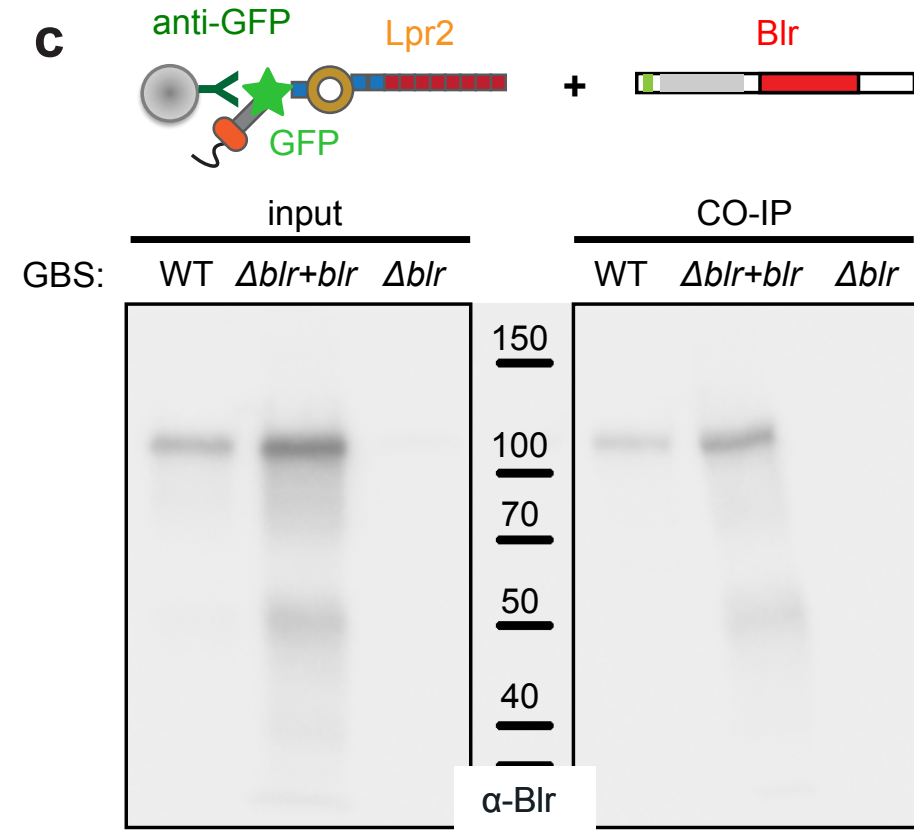

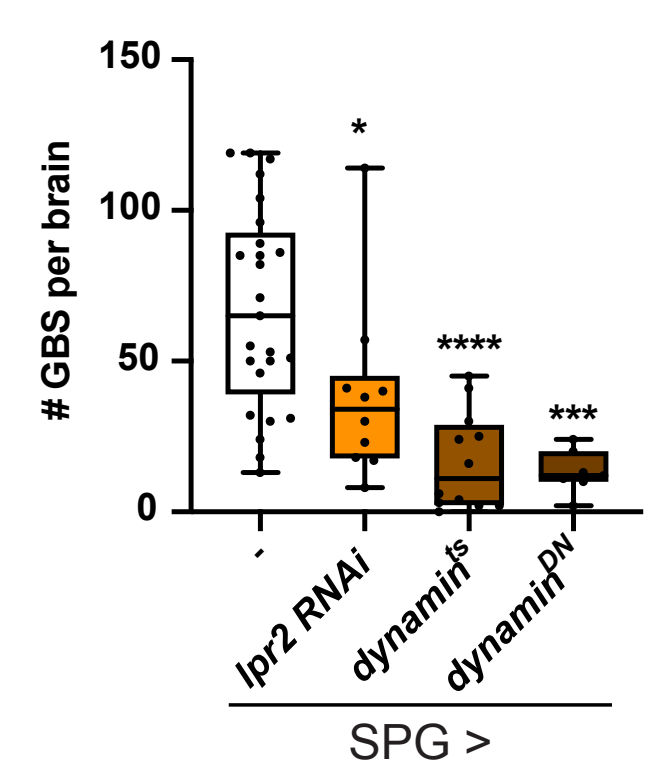

e
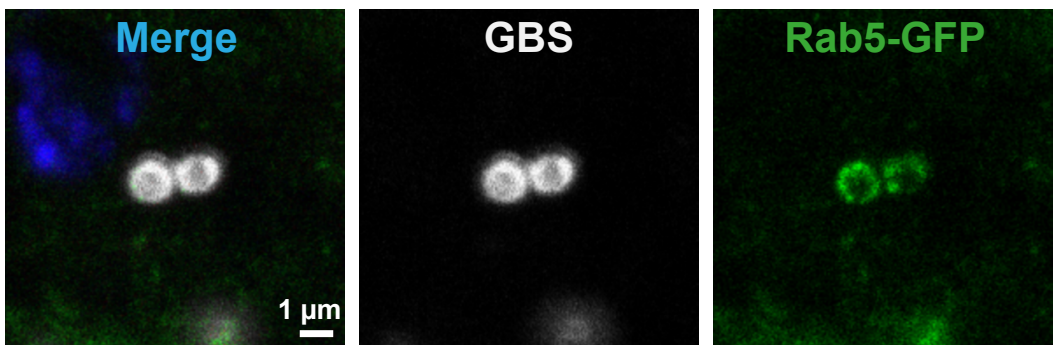

LpR2::GFP
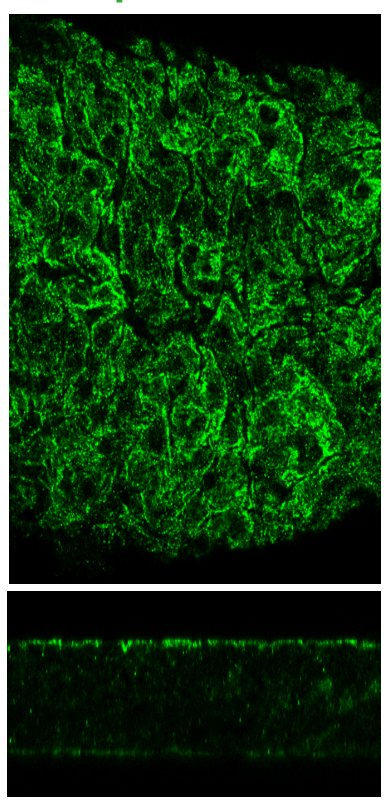

$\leftarrow \mathrm{B} / \mathrm{r}$

Merge

GBS

Lamp1-GFP

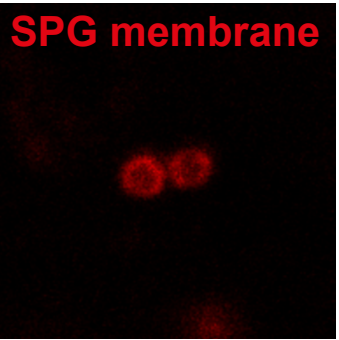

Lysosome
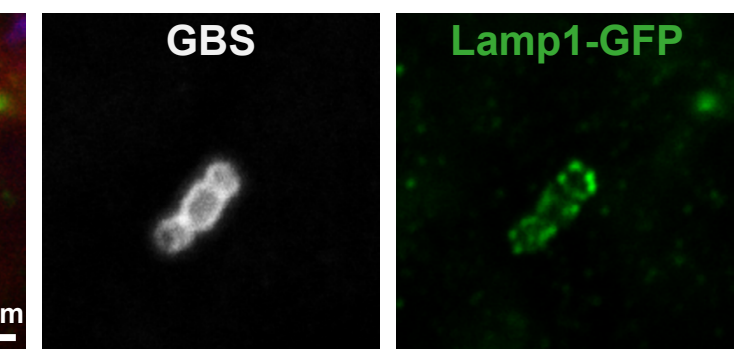

membrane

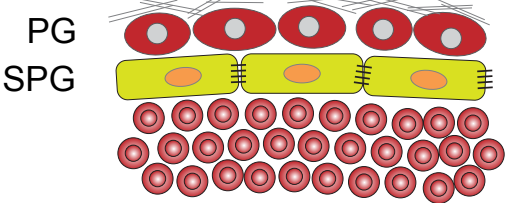


Figure 5

a

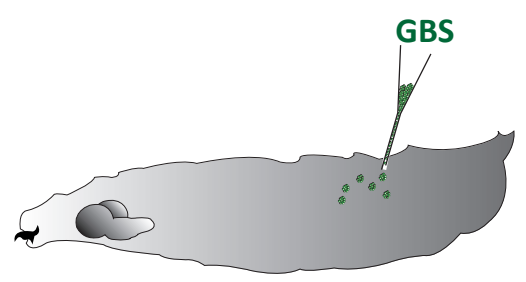

GBS entry

$4 \mathrm{~h}$ post-injection

b

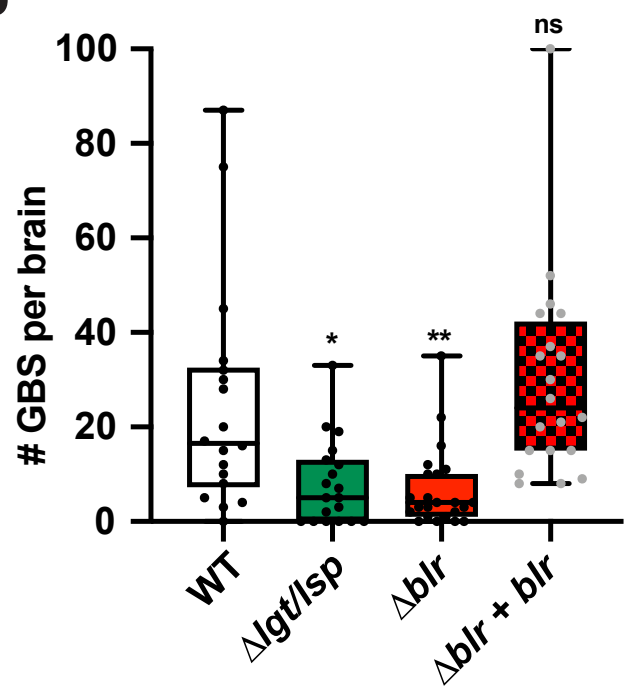

d

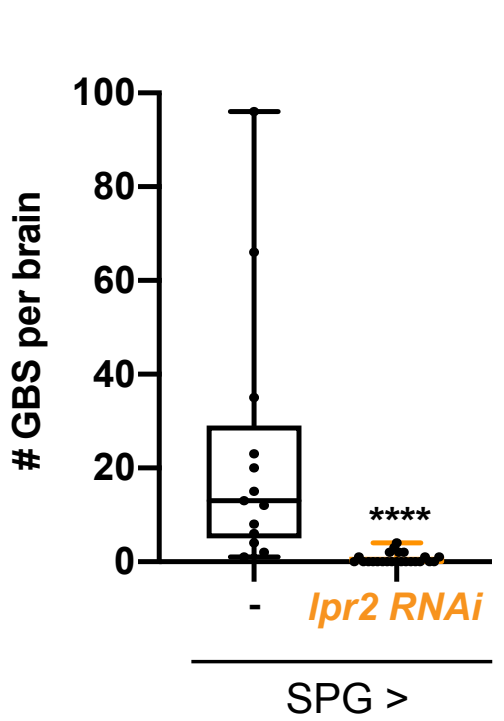

Inside
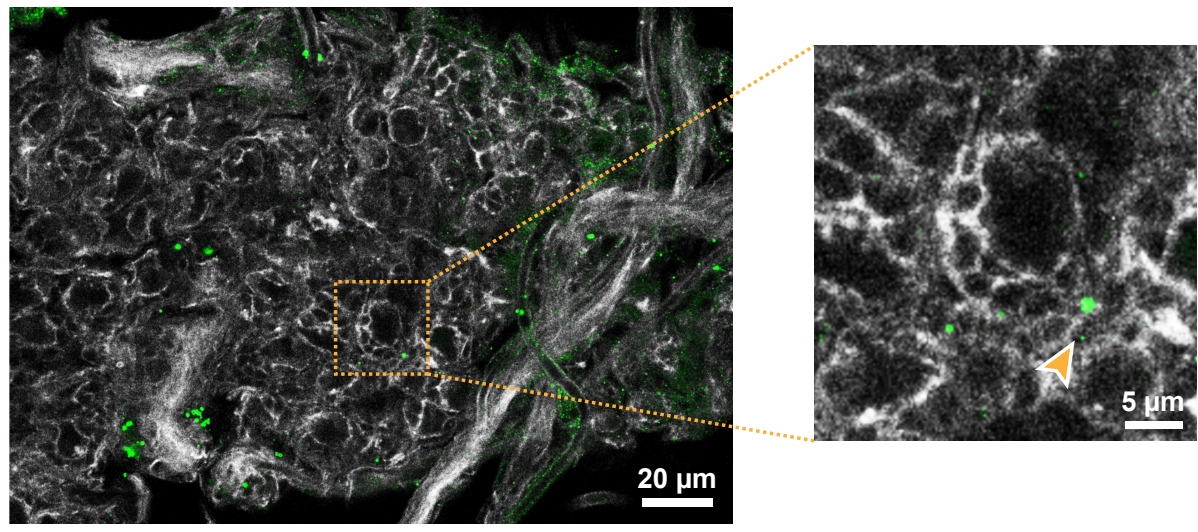

GBS Actin

Survival
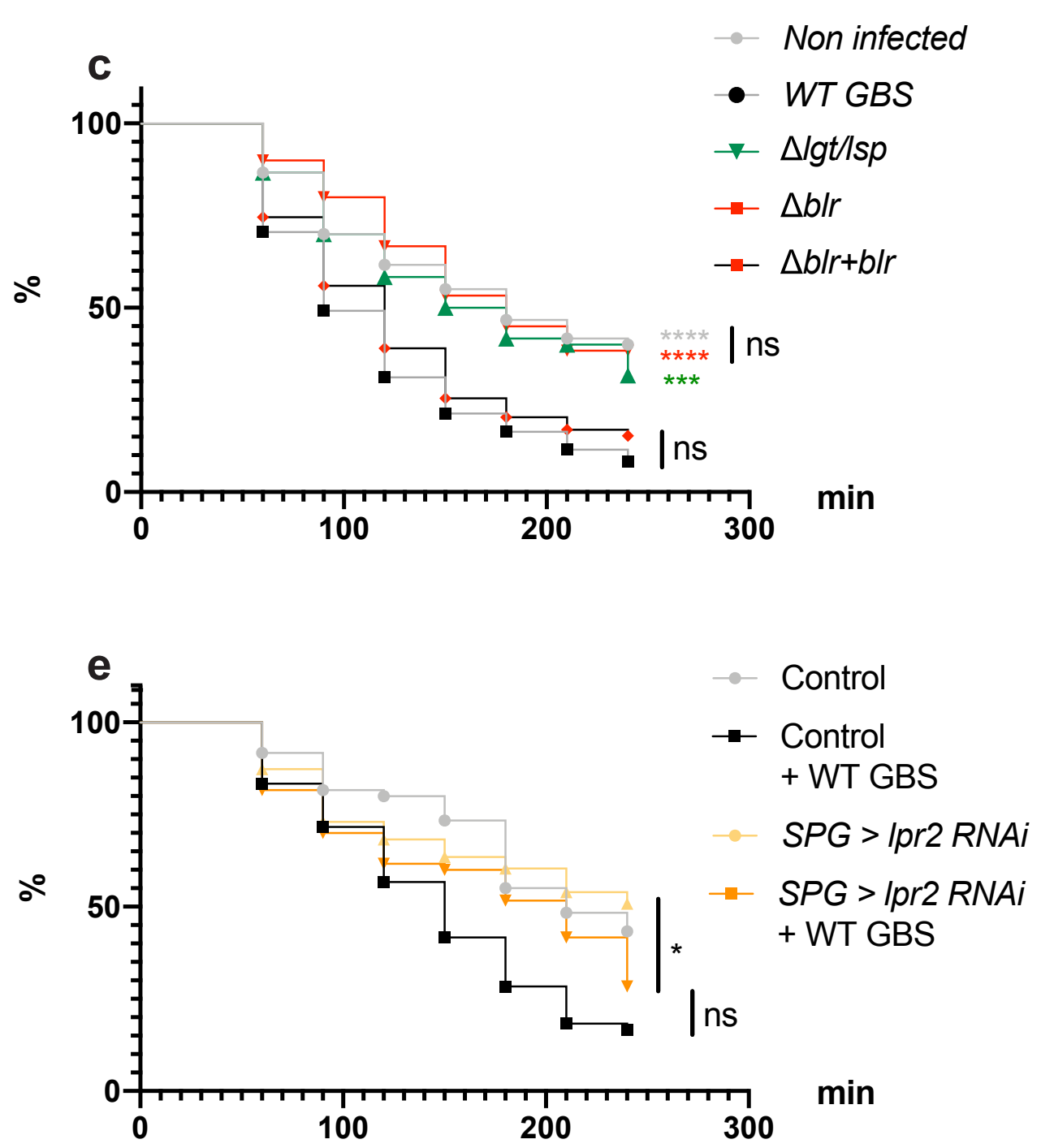
Figure 6
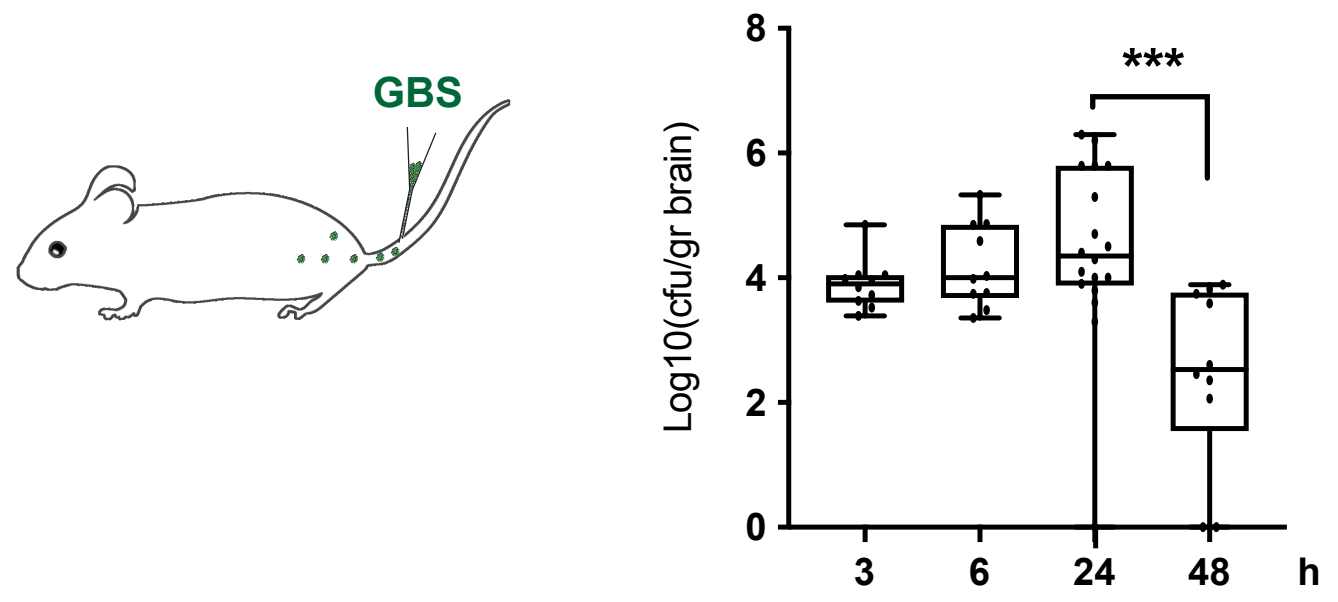

b $\quad$ GBS count in the blood

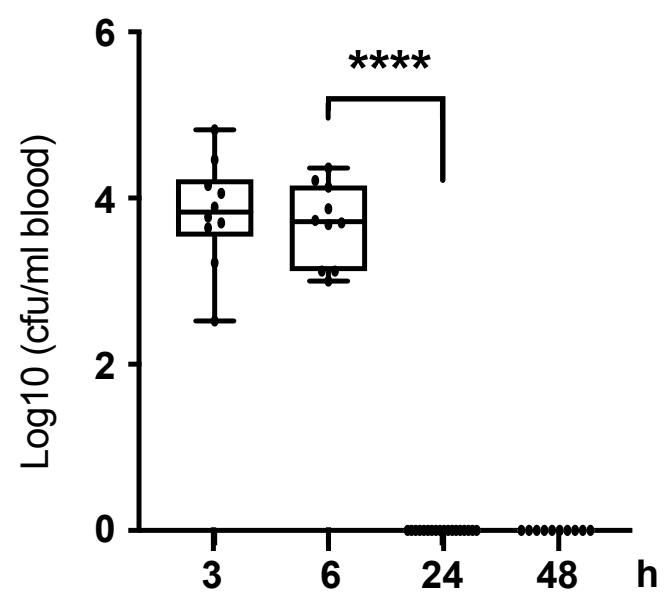

GBS-GFP $4 \mathrm{~h}$ post-infection

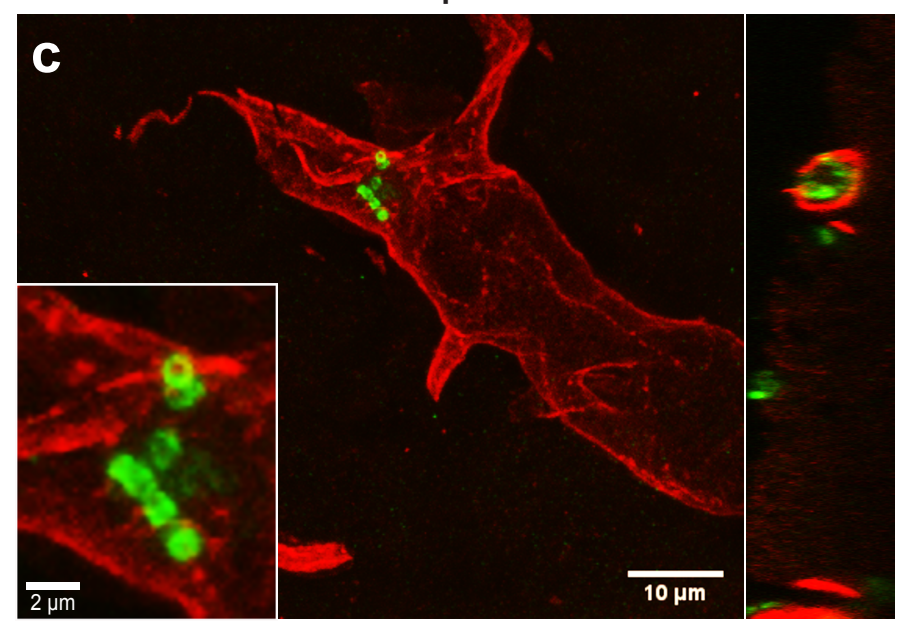

GFP CD31

GBS-GFP 24 h post-infection
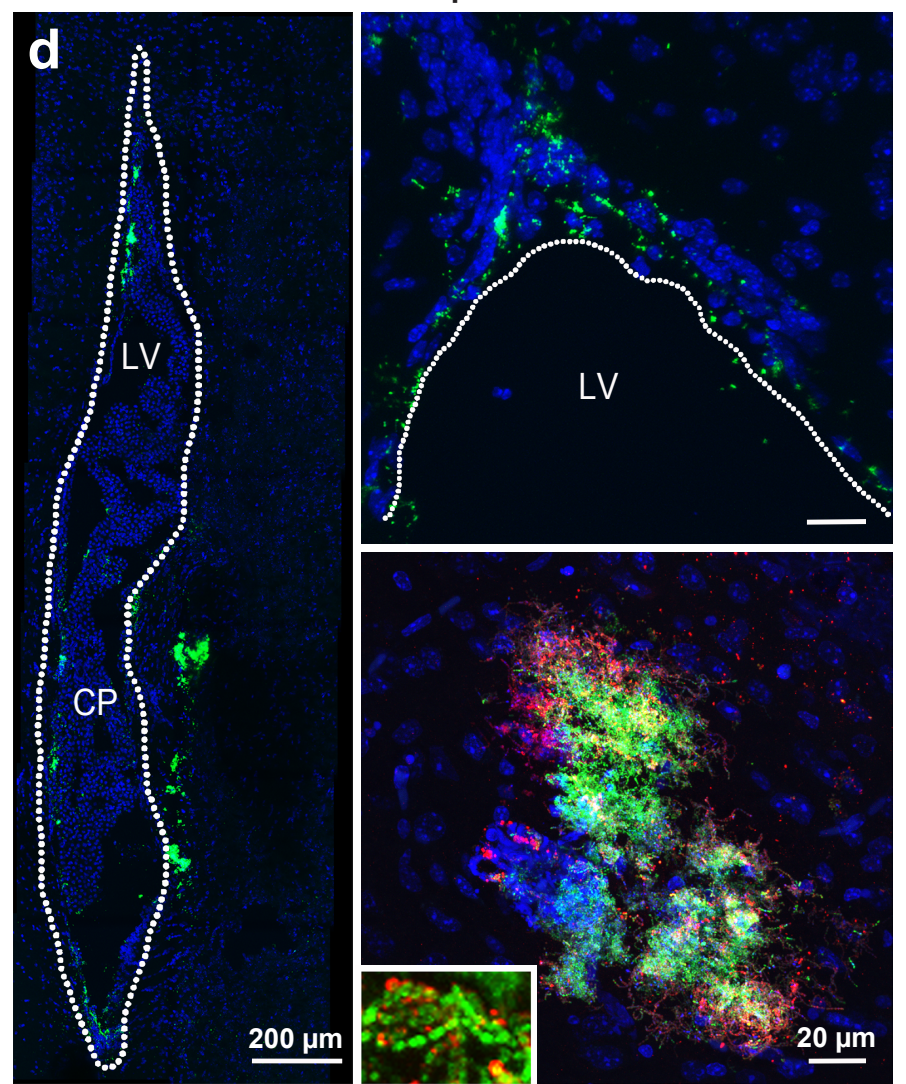

e

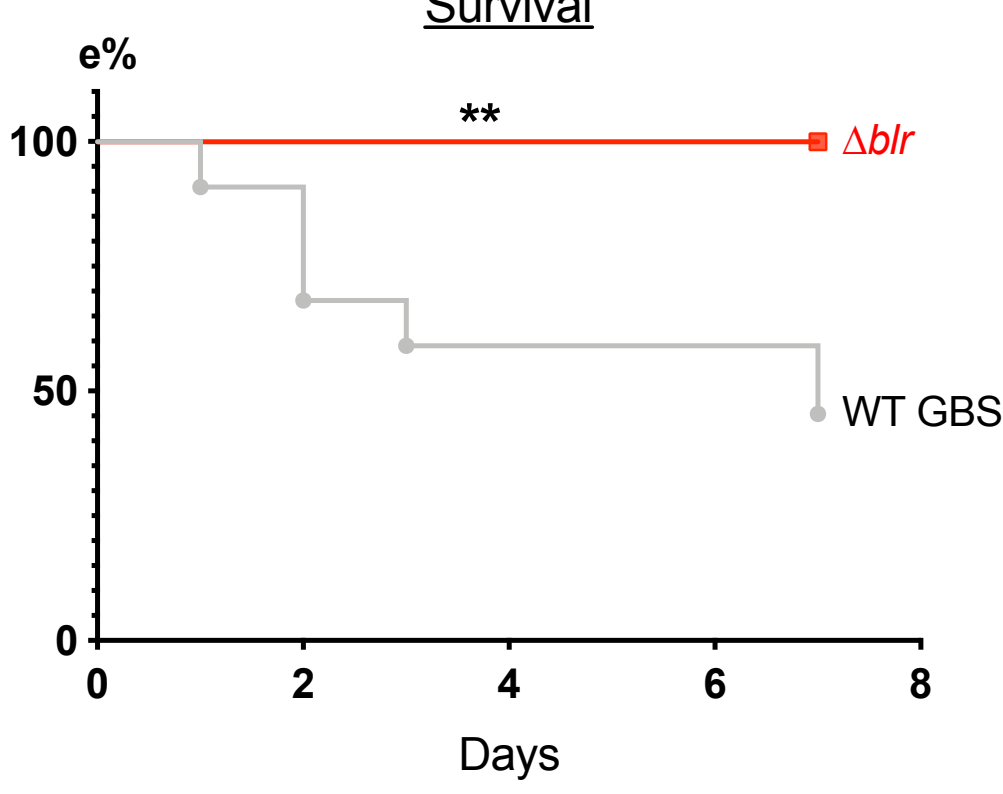

f

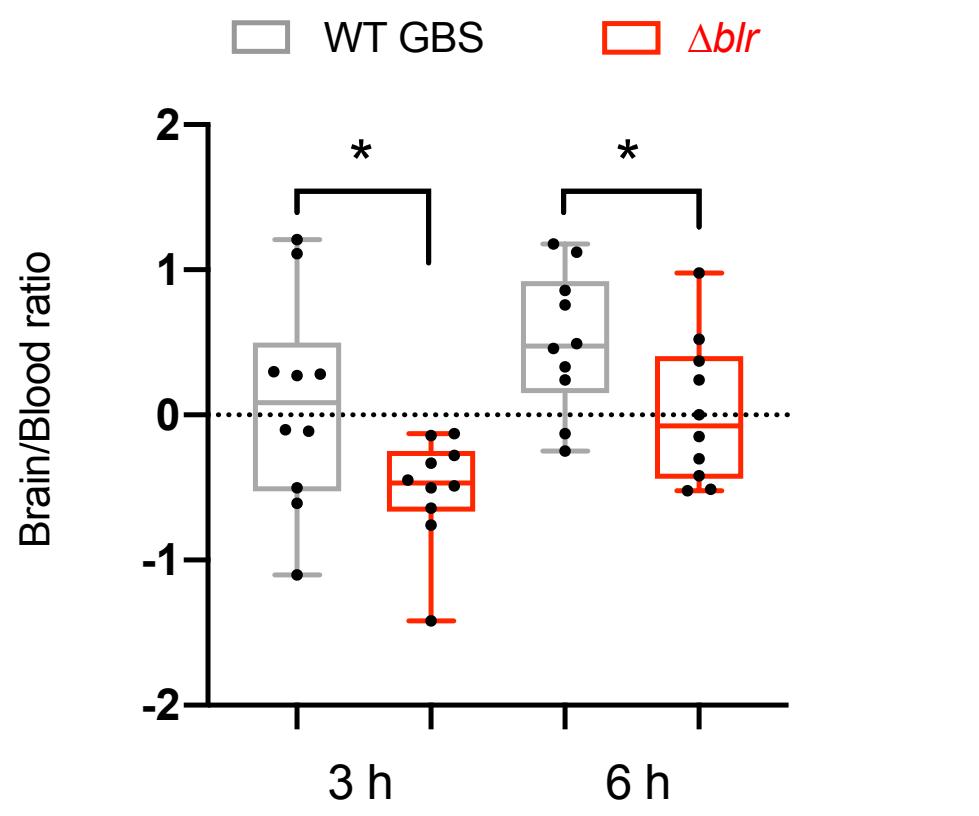


